\title{
USE OF GRAPHICAL METHODS IN THE DIAGNOSTIC OF PARAMETRIC PROBABILITY DISTRIBUTIONS FOR BIVARIATE LIFETIME DATA IN PRESENCE OF CENSORED DATA
}

\author{
Jorge Alberto Achcar ${ }^{1}$, Jose Rafael Tovar Cuevas ${ }^{2}$, Fernando A. Moala ${ }^{3 *}$ \\ ${ }^{1}$ Department of Social Medicine, University of Sao Paulo, Ribeirao Preto, Brazil. \\ ${ }^{2}$ Department of Statistics, Universidad del Valle, Calli, Colombia. \\ ${ }^{3}$ Department of Statistics, UNESP - S ao Paulo State University, Brazil.
}

\begin{abstract}
The choice of an appropriate bivariate parametrical probability distribution for pairs of lifetime data in presence of censored observations usually is not a simple task in many applications. Each existing bivariate lifetime probability distribution proposed in the literature has different dependence structure. Commonly existing classical or Bayesian discrimination methods could be used to discriminate the best among different proposed distributions, but these techniques could not be appropriate to say that we have good fit of some particular model to the data set. In this paper, we explore a recent dependence measure for bivariate data introduced in the literature to propose a graphical and simple criterion to choose an appropriate bivariate lifetime distribution for data in presence of censored data.
\end{abstract}

Keywords: bivariate lifetime, Bayesian approach, censoring data, copula functions, diagnostic discrimination methods.

\footnotetext{
* Corresponding author

Email: f.moala@unesp.br
} 


\section{Introduction}

In many different studies such as in medicine, engineering, economy, ecology, among several others, we have bivariate data that usually have some dependence structure (see for example, Davarzani et al. (2015) and Icuma et al (2016). To model this dependence there are many statistical models introduced in the literature, as for example, frailty models and copula function models (see for example, Goethals et al., 2008). In lifetime data applications, we usually have the presence of censored data (see for example, Wang (2011), Martinez et al. (2016) and Karvanen et al (2010). In the analysis of right censored univariate lifetime data, the non-parametric product-limit estimator proposed by Kaplan and Meier (1958) for the survival function $\mathrm{S}(\mathrm{t})=\mathrm{P}(\mathrm{T}>\mathrm{t})$, where $\mathrm{T}$ is a random variable denoting a lifetime and $\mathrm{t}$ is a fixed value, usually is the first step in any lifetime statistical analysis, as in medical or engineering applications (see for example, Lawless,1982; or Klein and Moeschberger,1995). In this situation, it is common the use of graphical methods to choose an appropriate parametrical distribution by comparing the fitted survival function with plots of the KaplanMeier product-limit estimates for the survival function. For the situation of bivariate lifetimes $\mathrm{T}_{1}$ and $\mathrm{T}_{2}$ in presence of censored observations, different nonparametric estimators for the joint survival function $S\left(t_{1}, t_{2}\right)=P\left(T_{1}>t_{1}, T_{2}>t_{2}\right)$ were proposed in the literature (see for example, Campbell, 1981; Campbell and Foldes, 1982; Langberg and Shaked, 1982; Hanley and Parnes, 1983; Tsai, Leurgans and Crowley, 1986; Dabrowska, 1988; Prentice and Cai, 1992 or Puritt, 1993).

In general, the nonparametric estimates for the bivariate survival function introduced in the literature have great complexities to be used in practical situations.

A special case that occurs in some applications is given when the two lifetimes are subject to independent censorship by a single censoring variable (univariate censorship).

In this situation, Lin and Ying (1993) introduced a nonparametric estimator of the bivariate survival function in presence of censored data with nice statistical properties (strong consistency, weakly convergence to zero-mean Gaussian process) that is simple to be applied for bivariate lifetime data analysis in presence of univariate censoring.

In this paper, it is proposed the use of the Lin-Ying nonparametric estimator for the joint survival function in presence of univariate censorship in a new graphical diagnostic approch to compare different parametrical bivariate lifetime models exploring a recent dependence measure introduced by Ledwina (2015) for two random variables.

In this new graphical methodology, the LY nonparametric estimator of the joint survival function $S\left(t_{1}, t_{2}\right)$ in presence of univariate censoring and the Kaplan and Meier nonparametric estimators for the marginal survival functions $S_{1}\left(\mathrm{t}_{1}\right)$ and $S_{2}\left(\mathrm{t}_{2}\right)$ are used in the Ledwina dependence measure.

Three bivariate lifetime parametric models are considered in this study in presence of censored data: the Block and Basu (1974) exponential distribution and two bivariate Weibull distributions derived from copula functions (see for example, Flores, 2016). The main goal of this paper is to introduce a simple graphical method to choose an appropriate bivariate lifetime parametrical distribution based on plots of the empirical Ledwina measure of dependence.

The paper is organized as follows: in section 2, it is presented the Lin and Ying estimator; in section 3, it is introduced the Ledwina dependence measure; in section 4, it is introduced the Block and Basu bivariate distribution; in section 5, it is introduced some special copula 
functions; section 6 presents five applications; finally section 7 presents some concluding remark.

\section{The Lin and Ying estimator}

The Lin-Ying (1993) non-parametric estimator simply denoted as the LY estimator for the joint survival function is based on the idea that the bivariate survival function of paired survival times can be expressed as the ratio of the bivariate at-risk probability to the survival function of the censoring time.

The LY estimator of the joint survival function $S\left(t_{1}, t_{2}\right)$ in presence of univariate censoring is given as follows: let $\left(T_{1 \mathrm{i}}, T_{2 \mathrm{i}}\right), i=1,2, \ldots, n$ be bivariate pairs of lifetimes and $\mathrm{C}_{\mathrm{i}}, \mathrm{i}=1, \ldots$, $\mathrm{n}$ be identically distributed censoring times with survival function $G(c)=P(C>c)$. Define the indicator variables,

$$
{ }_{1 i}=\left\{\begin{array}{ll}
1 & \text { if } T_{1 i} \leq C_{i} \\
0 & \text { if } T_{1 i}>C_{i}
\end{array} \text { and }{ }_{2 i}= \begin{cases}1 & \text { if } T_{2 i} \leq C_{i} \\
0 & \text { if } T_{2 i}>C_{i}\end{cases}\right.
$$

Also define, $U_{i}=\min \left(T_{1 i}, C_{i}\right)$ and $V_{i}=\min \left(T_{2 i}, C_{i}\right), i=1,2, \ldots, n$. In this way, a nonparametric estimator of $S\left(t_{1}, t_{2}\right)=P\left(T_{1}>\mathrm{t}_{1}, T_{2}>t_{2}\right)=\frac{P\left(U>t_{1}, V>t_{2}\right)}{G(\mathrm{z})}$ where $z=$ $\max \left(t_{1}, t_{2}\right)$ is obtained using the following results:

(i) $P_{n}\left(U>t_{1}, V>t_{2}\right)=\frac{\sum_{i=1}^{n} I\left(U>t_{1}, V>t_{2}\right)}{n}$ (empirical survival function )

(ii) To get an estimator of $G(\cdot)$ it is observed that in this case the data are given by $\left(R_{i}, \delta c_{i}\right)$, $i=1,2, \ldots, n$ where, $R_{i}=\min \left(C_{i}, Z_{i}\right), Z_{i}=\max \left(T_{1 i}, T_{2 i}\right)$ or $R_{i}=\max \left(U_{i}, V_{i}\right)$, and $\delta c_{i}=I\left[C_{i} \leq Z_{\mathrm{i}}\right]=1-\left(\delta_{1 i}\right)\left(\delta_{2 i}\right)$.

In this case it is used the Kaplan-Meier product-limit estimator. Let $\left\{c_{k} ; k \geq 1\right\}$ be the ordered sequence of distinct time points where the censoring occurs, and let $d_{k}$ be the number of censored observations, at $c_{k}$. Also, let $n_{k}=\sum_{i=1}^{n} I\left(R_{i} \geq c_{k}\right)$ Thus,

$$
\hat{G}(t)=\prod_{k: c_{k}<t} \frac{n_{k}-d_{k}}{n_{k}}
$$

(iii) In this way, the LY estimator of the joint survival function is given by,

$$
\hat{S}\left(t_{1}, t_{2}\right)=P\left(T_{1}>t_{1}, T_{2}>t_{2}\right)=\frac{\sum_{i=1}^{n} I\left(U_{i}>t_{1}, V_{i}>t_{2}\right)}{n \hat{G}(z)}
$$

where $z=\max \left(t_{1}, t_{2}\right)$.

Some remarks:

(i) The LY estimator is simpler than any other nonparametric estimator for the bivariate survival function introduced in the literature and it is reduced to the usual empirical survival function in the absence of censoring.

(ii) The LY estimator (2.3)is a natural generalization of the univariate productlimit (Kaplan-Meier) estimator. 
(iii) From (2.3) it is possible to get a nonparametric estimator for the joint cumulative distribution function from the result, $F\left(t_{1}, t_{2}\right)=P\left(T_{1} \leq t_{1}, T_{2} \leq t_{2}\right)=1-$ $\left(P\left(T_{1}>t_{1}\right)+P\left(T_{2}>t_{2}\right)\right)+P\left(T_{1}>t_{1}, T_{2}>t_{2}\right)$.

\section{The Ledwina dependence measure}

It is important to point out that in applications usually there are great difficulties to decide by an appropriate bivariate lifetime model which adequately models the strength of dependence between two random variables. Dependence scalar measure indexes or global measures of dependence for two random variables were studied by many authors (see for example, Jogdeo, 1982; Lancaster, 1982; Drouet and Kotz, 2001; Balakrishnan and Lai, 2009). Usually the use of scalar dependence bivariate measures could be not the best way to represent complex dependence structures and other dependence measures were introduced in the literature, as local indexes (see, for example, Drouet and Kotz, 2001; Kowalczyk and Pleszczynska, 1997; Bjerve and Doksum, 1993 and Bairamov et al., 2003).

In a recent paper, Ledwina (2015) introduced a new function valued measure of dependence of two random variables $T_{1}$ and $T_{2}$ and presented the main properties of this measure. The proposed measure has simple form and its definition explores only cumulative distribution functions.

The measure takes values in the interval $[-1,1]$ and treats both variables in a symmetrical way. The measure preserves the correlation order, or equivalently the concordance order, which is the quadrant order restricted to the class of distributions with fixed marginals.

The Ledwina dependence measure in the general case assuming two random variables $T_{1}$ and $T_{2}$ is given by,

$$
q\left(t_{1}, t_{2}\right)=\frac{F\left(t_{1}, t_{2}\right)-F_{1}\left(t_{1}\right) F_{2}\left(t_{2}\right)}{w\left(t_{1}, t_{2}\right)}, \text { for }\left(t_{1}, t_{2}\right) \in D
$$

where $\omega\left(t_{1}, t_{2}\right)=\left[F_{1}\left(t_{1}\right) F_{2}\left(t_{2}\right)\left(1-F_{1}\left(t_{1}\right)\right)\left(1-F_{2}\left(t_{2}\right)\right)\right]^{\frac{1}{2}}, D=\left\{\left(t_{1}, t_{2}\right), 0<F_{1}\left(t_{1}\right)<\right.$ $\left.1,0<F_{2}\left(t_{2}\right)<1\right\}, F_{1}\left(t_{1}\right)$ and $F_{2}\left(t_{2}\right)$ are the marginal distribution functions for $T_{1}$ and $T_{2}$, that is , $F_{1}\left(t_{1}\right)=P\left(T_{1} \leq t_{1}\right), F_{2}\left(t_{2}\right)=P\left(T_{2} \leq t_{2}\right) ; \mathrm{F}\left(t_{1}, t_{2}\right)$ is the joint distribution function for $T_{1}$ and $T_{2}$ given by $\mathrm{F}\left(t_{1}, t_{2}\right)=P\left(T_{1} \leq t_{1}, T_{2} \leq t_{2}\right)$.

From (3.1)it is seen that $q$ treats both variables $T_{1}$ and $T_{2}$ symmetrically and a knowledge of $q$ and the marginal distributions allows one to recover the joint distribution function for $T_{1}$ and $T_{2}$ (for properties of $q$ and more details, see Ledwina, 2015). Observe that $-1<$ $q\left(t_{1}, t_{2}\right)<1$.

An empirical simple estimator for the Ledwina dependence (3.1) considering the bivariate data set $\left(t_{1 \mathrm{i}}, t_{2 \mathrm{i}}\right), i=1,2, \ldots, n$ not in the presence of censored data is obtained replacing $\mathrm{F}\left(t_{1}, t_{2}\right), F_{1}\left(t_{1}\right)$ and $F_{2}\left(t_{2}\right)$ by their respective empirical estimators, 


$$
\begin{aligned}
& F_{n}\left(t_{1}, t_{2}\right)=\frac{\sum_{i=1}^{n} I\left(T_{1} \leq t_{1}, T_{2} \leq t_{2}\right)}{n} \\
& F_{n}\left(t_{1}\right)=\frac{\sum_{i=1}^{n} I\left(T_{1} \leq t_{1}\right)}{n} \\
& F_{n}\left(t_{2}\right)=\frac{\sum_{i=1}^{n} I\left(T_{2} \leq t_{2}\right)}{n}
\end{aligned}
$$

for fixed values $t_{1}$ and $t_{1}$, where $I(\cdot)$ is the indicator function.

Similarly, we could consider the survival functions $\mathrm{S}\left(t_{1}, t_{2}\right), S_{1}\left(t_{1}\right)$ and $S_{2}\left(t_{2}\right)$ in place of $F\left(t_{1}, t_{2}\right), F_{1}\left(t_{1}\right)$ and $F_{2}\left(t_{2}\right)$ in (3.1) obtaining a dependence measure given by,

$$
r\left(t_{1}, t_{2}\right)=\frac{S\left(t_{1}, t_{2}\right)-S_{1}\left(t_{1}\right) S_{2}\left(t_{2}\right)}{w\left(t_{1}, t_{2}\right)}, \text { for }\left(t_{1}, t_{2}\right) \in D
$$

where $\omega\left(t_{1}, t_{2}\right)=\left[S_{1}\left(t_{1}\right) S_{2}\left(t_{2}\right)\left(1-S_{1}\left(t_{1}\right)\right)\left(1-S_{2}\left(t_{2}\right)\right)\right]^{\frac{1}{2}}, D=\left\{\left(t_{1}, t_{2}\right), 0<S_{1}\left(t_{1}\right)<\right.$ $\left.1,0<S_{2}\left(t_{2}\right)<1\right\}$.

An empirical estimator of $\mathrm{r}\left(t_{1}, t_{2}\right)$ is given replacing $\mathrm{S}\left(t_{1}, t_{2}\right), S_{1}\left(t_{1}\right)$ and $S_{2}\left(t_{2}\right)$ by the empirical estimators,

$$
\begin{gathered}
S_{n}\left(t_{1}, t_{2}\right)=\frac{\sum_{i=1}^{n} I\left(T_{1}>t_{1}, T_{2}>t_{2}\right)}{n} \\
S_{n}\left(t_{1}\right)=\frac{\sum_{i=1}^{n} I\left(T_{1}>t_{1}\right)}{n} \\
S_{n}\left(t_{2}\right)=\frac{\sum_{i=1}^{n} I\left(T_{2}>t_{2}\right)}{n}
\end{gathered}
$$

\section{The Block and Basu distribution}

To analyse bivariate lifetimes it is possible to assume different parametric distributions introduced in the literature (see for example, Freund, 1961; Marshall and Olkin, 1967; Sarkar, 1987; Downton, 1970; Gumbel, 1960; Hawkes, 1972; Hougaard, 1986; Arnold and Strauss, 1988). Among all these bivariate lifetime distributions, one model has been very well explored in the literature: the Block and Basu (1974) bivariate exponential distribution, denoted as the BB distribution. The BB distribution with parameters, $\lambda_{1}, \lambda_{1}$ and $\lambda_{3}$ for the lifetimes $T_{1}$ and $T_{2}$ has a joint density function given by,

$$
f\left(t_{1}, t_{2}\right)=\left\{\begin{array}{lll}
\frac{123}{12} \exp \left\{{ }_{1} t_{1}\right. & \left.{ }_{23} t_{2}\right\} & \text { if } t_{1}<t_{2} \\
\frac{{ }_{13}}{{ }_{12}} \exp \left\{{ }_{13} t_{1}\right. & \left.{ }_{2} t_{2}\right\} & \text { if } t_{1} \geq t_{2}
\end{array}\right.
$$


where $\lambda_{12}=\lambda_{1}+\lambda_{2}, \lambda_{13}=\lambda_{1}+\lambda_{3}, \lambda_{23}=\lambda_{2}+\lambda_{3}$ and $\lambda=\lambda_{1}+\lambda_{2}+\lambda_{3}$.

The joint survival function for the BB distribution is given by,

$$
S\left(t_{1}, t_{2}\right)=P\left\{T_{1}>t_{1}, T_{2}>t_{2}\right\}= \begin{cases}S_{1}\left(t_{1}, t_{2}\right) & \text { if } t_{1}<t_{2} \\ S_{2}\left(t_{1}, t_{2}\right) & \text { if } t_{1}>t_{2}\end{cases}
$$

where

$$
S_{1}\left(t_{1}, t_{2}\right)=\frac{\lambda}{\lambda_{12}} \exp \left\{-\lambda_{1} t_{1}-\lambda_{23} t_{2}\right\}-\frac{\lambda_{3}}{\lambda_{12}} \exp \left\{-\lambda_{2} t_{2}\right\}
$$

and

$$
S_{2}\left(t_{1}, t_{2}\right)=\frac{\lambda}{\lambda_{12}} \exp \left\{-\lambda_{13} t_{1}-\lambda_{2} t_{2}\right\}-\frac{\lambda_{3}}{\lambda_{12}} \exp \left\{-\lambda_{1} t_{1}\right\}
$$

Considering the $\mathrm{BB}$ bivariate exponential distribution, the marginal distributions for $T_{1}$ and $T_{2}$ are mixtures of exponentials having an absolutely continuous joint distribution with positive correlation coefficient. Bayesian analysis for bivariate lifetime distributions has been considered as a good alternative to get the inferences of interest when compared to the use of standard classical inference methods, in terms of better accuracy for the obtained inferences of interest. Bayesian inference is based on the Bayes formula, where the posterior distribution is obtained combining the likelihood function with the prior distributions for the parameters of the model. With the use of existing simulation methods, it is possible to get accurate Monte Carlo estimates for all parameters of interest. In this way, the use of MCMC (Markov Chain Monte Carlo) methods (see for example, Gelfand and Smith, 1990 or Chib and Greenberg, 1995) gives a good alternative to obtain accurate posterior summaries of interest.

Bayesian approaches for the BB lifetime distribution using MCMC methods in presence or not of covariates and censored data are presented by many authors (see for example, Achcar and Leandro, 1998; or Achcar and Santos, 2011).

\subsection{The likelihood function}

Suppose either $T_{1}$ or $T_{2}$ can be censored and that censoring is independent of the lifetimes. Let us subdivide the $n$ observations into four cases:

$C_{1}$ : both $t_{1 i}$ and $t_{2 i}$ are observed lifetimes, $i=1,2, \ldots, n$;

$C_{2} t_{1 i}$ is a lifetime and $t_{2 i}$ is a censoring time (that is, we only know that $T_{2 \mathrm{i}} \geq t_{2 i}$ );

$C_{3}: t_{1 i}$ is a censoring time and $t_{2 i}$ is a lifetime;

$C_{4}$ : both $t_{1 i}$ and $t_{2 i}$ are censoring times.

The likelihood function for a continuous model (see for example, Lawless, 1982, page 479) is given by,

$$
L=\prod_{i \in C_{1}} f\left(t_{1 i}, t_{2 i}\right) \prod_{i \in C_{2}}\left(-\frac{\partial S\left(t_{1 i}, t_{2 i}\right)}{\partial t_{1 i}}\right) \prod_{i \in C_{3}}\left(-\frac{\partial S\left(t_{1 i}, t_{2 i}\right)}{\partial t_{2 i}}\right) \prod_{i \in C_{4}} S\left(t_{1 i}, t_{2 i}\right)
$$

Assuming the BB bivariate exponential distribution with density function (4.1) and survival function (4.2), we have, 


$$
\frac{\partial S\left(t_{1 i}, t_{2 i}\right)}{\partial t_{1 i}}=\left\{\begin{array}{cc}
S_{1 t_{1}}^{\prime}\left(t_{1 i}, t_{2 i}\right) & \text { if } t_{1 i}<t_{2 i} \\
S_{2 t_{1}}^{\prime}\left(t_{1 i}, t_{2 i}\right) & \text { if } t_{1 i} \geq t_{2 i}
\end{array}\right.
$$

where

$$
S_{1 t_{1}}^{\prime}\left(t_{1 i}, t_{2 i}\right)=\frac{\lambda \lambda_{1}}{\lambda_{12}} \exp \left\{-\lambda_{1} t_{1 i}-\lambda_{23} t_{2 i}\right\}
$$

and

$$
S_{2 t_{1}}^{\prime}\left(t_{1 i}, t_{2 i}\right)=\frac{\lambda \lambda_{13}}{\lambda_{12}} \exp \left\{-\lambda_{13} t_{1 i}-\lambda_{2} t_{2 i}\right\}-\frac{\lambda \lambda_{3}}{\lambda_{12}} \exp \left\{-\lambda_{1} t_{1 i}\right\}
$$

Also,

$$
\frac{\partial S\left(t_{1 i}, t_{2 i}\right)}{\partial t_{2 i}}= \begin{cases}S_{1 t_{2}}^{\prime}\left(t_{1 i}, t_{2 i}\right) & \text { if } t_{1 i}<t_{2 i} \\ S_{2 t_{2}}^{\prime}\left(t_{1 i}, t_{2 i}\right) & \text { if } t_{1 i} \geq t_{2 i}\end{cases}
$$

where

$$
S_{1 t_{2}}^{\prime}\left(t_{1 i}, t_{2 i}\right)=\frac{\lambda \lambda_{23}}{\lambda_{12}} \exp \left\{-\lambda_{1} t_{1 i}-\lambda_{23} t_{2 i}\right\}-\frac{\lambda \lambda_{3}}{\lambda_{12}} \exp \left\{-\lambda_{2} t_{2 i}\right\}
$$

and

$$
S_{2 t_{2}}^{\prime}\left(t_{1 i}, t_{2 i}\right)=\frac{\lambda \lambda_{2}}{\lambda_{12}} \exp \left\{-\lambda_{13} t_{1 i}-\lambda_{2} t_{2 i}\right\}
$$

Let us define the indicator variables $\delta_{1 i}, \delta_{2 i}$ and $v_{i}$, by,

$$
{ }_{j i}= \begin{cases}1 & \text { if } t_{j i} \text { is an observed lifetime } \\ 0 & \text { if } t_{j i} \text { is a censored observation }\end{cases}
$$

for $j=1,2 ; i=1, \ldots, n$ and

$$
v_{i}= \begin{cases}1 & \text { if } t_{1 i}<t_{2 i} \\ 0 & \text { if } t_{1 i} \geq t_{2 i}\end{cases}
$$

In this way, the log-likelihood function for $\lambda_{1}, \lambda_{2}$ and $\lambda_{3}$ is given by,

$$
\begin{gathered}
l\left(\lambda_{1}, \lambda_{2}, \lambda_{3}\right)=\log L\left(\lambda_{1}, \lambda_{2}, \lambda_{3}\right)=\sum_{i=1}^{n} v_{i} \delta_{1 i} \delta_{2 i} \log f_{1}\left(t_{1 i}, t_{2 i}\right)+\sum_{i=1}^{n}\left(1-v_{i}\right) \delta_{1 i} \delta_{2 i} \operatorname{iog} \\
f_{2}\left(t_{1 i}, t_{2 i}\right)+\sum_{i=1}^{n} v_{i} \delta_{1 i}\left(1-\delta_{2 i}\right) \log S_{1 t_{1}}^{\prime}\left(t_{1 i}, t_{2 i}\right)+\sum_{i=1}^{n}\left(1-v_{i}\right) \delta_{1 i}\left(1-\delta_{2 i}\right) \log S_{2 t_{1}}^{\prime}\left(t_{1 i}, t_{2 i}\right)+ \\
\sum_{i=1}^{n} v_{i}\left(1-\delta_{1 i}\right) \delta_{2 i} \log S_{1 t_{2}}^{\prime}\left(t_{1 i}, t_{2 i}\right)+\sum_{i=1}^{n}\left(1-v_{i}\right)\left(1-\delta_{1 i}\right) \delta_{2 i} \log S_{2 t_{2}}^{\prime}\left(t_{1 i}, t_{2 i}\right)+\sum_{i=1}^{n} v_{i}\left(1-\delta_{1 i}\right) \\
\left(1-\delta_{2 i}\right) \log S_{1}\left(t_{1 i}, t_{2 i}\right)+\sum_{i=1}^{n}\left(1-v_{i}\right)\left(1-\delta_{1 i}\right)\left(1-\delta_{2 i}\right) \log S_{2}\left(t_{1 i}, t_{2 i}\right)
\end{gathered}
$$

For a Bayesian analysis of the BB distribution in the presence of censored observations, let us assume independent gamma distributions for the parameters $\lambda_{k}$, that is 


$$
\lambda_{k} \sim \operatorname{Gamma}\left[a_{k}, b_{k}\right]
$$

for $k=1,2,3 ; a_{k}$ and $b_{k}$ are known hyperparameters; Gamma $\left[a_{k}, b_{k}\right]$ denotes a gamma distribution with mean $\frac{a_{k}}{b_{k}}$ and variance $\frac{a_{k}}{b_{k}^{2}}$.

The joint posterior distribution for $\lambda_{1}, \lambda_{2}$ and $\lambda_{3}$ is given by,

$$
\pi\left(\lambda_{1}, \lambda_{2}, \lambda_{3} \mid t_{1}, t_{2}\right) \propto\left(\prod_{k=1}^{3} \lambda_{k}^{\mathrm{a}_{k}-1} e^{-b_{k} \lambda_{k}}\right) L\left(\lambda_{1}, \lambda_{2}, \lambda_{3}\right)
$$

where $L\left(\lambda_{1}, \lambda_{2}, \lambda_{3}\right)$ is the likelihood function and $t_{1}$ and $t_{2}$ are the vectors of observed data.

To simulate samples for joint posterior distribution (4.10), it is used MCMC methods.

In this way, it is simulated samples for the joint posterior distribution (4.10), from the full posterior conditional distributions $\pi\left(\lambda_{1} \mid \lambda_{2}, \lambda_{3}, t_{1}, t_{2}\right), \pi\left(\lambda_{2} \mid \lambda_{1}, \lambda_{3}, t_{1}, t_{2}\right)$ and $\pi\left(\lambda_{3} \mid \lambda_{1}, \lambda_{2}\right.$, $\left.t_{1}, t_{2}\right)$ using the Gibbs sampling algorithm or the Metropolis-Hastings algorithm when the full posterior conditional distributions have unknown forms.

\section{Use of copula functions}

The use of copula functions (Sklar, 1959) is becoming very popular in the construction of new parametrical multivariate distributions (see for example, Durante and Sempi, 2015 or Nelsen, 2006). Copula functions can be used to link marginal distributions with a joint distribution.

For specified univariate marginal distribution functions $F_{1}\left(t_{1}\right), F_{2}\left(t_{2}\right), \ldots, F_{\mathrm{m}}\left(t_{\mathrm{m}}\right)$, the function,

$$
C\left(F_{1}\left(t_{1}\right), F_{2}\left(t_{2}\right), \ldots, F_{m}\left(t_{m}\right)\right)=F\left(t_{1}, t_{2}, \ldots, t_{m}\right)
$$

which is defined using a copula function $C$, results in a multivariate distribution function with univariate marginal distributions specified as $F_{1}\left(t_{1}\right), F_{2}\left(t_{2}\right), \ldots, F_{\mathrm{m}}\left(t_{\mathrm{m}}\right)$.

It is important to point out that any multivariate distribution function $F$ can be written in the form of a copula function (Sklar, 1959); that is, if $F\left(t_{1}, t_{2}, \ldots, t_{m}\right)$ is a joint multivariate distribution function with univariate marginal distribution functions $F_{1}\left(t_{1}\right), F_{2}\left(t_{2}\right), \ldots, F_{\mathrm{m}}\left(t_{\mathrm{m}}\right)$, thus there exists a copula function $C\left(U_{1}, U_{2}, \ldots, U_{m}\right)$ such that,

$$
F\left(t_{1}, t_{2}, \ldots, t_{m}\right)=C\left(F_{1}\left(t_{1}\right), F_{2}\left(t_{2}\right), \ldots, F_{m}\left(t_{m}\right)\right)
$$

If every $F_{i}$ is continuous, then $C$ is unique. For the special case of bivariate distributions, we have $m=2$.

The approach to formulate a multivariate distribution using a copula is based on the idea that a simple transformation can be made of each marginal variable in such a way that each transformed marginal variable has an uniform distribution. Once this is done, the dependence structure can be expressed as a multivariate distribution on the obtained uniforms, and a copula is precisely a multivariate distribution on marginally uniform random variables.

In this way, there are many families of copulas which differ in terms of the dependence structures.

In the bivariate case, let $T_{1}$ and $T_{2}$ be two random variables with continuous distribution functions $F_{1}$ and $F_{2}$. The probability integral transform can be applied separately to the two random variables to define $U_{1}=F_{1}\left(t_{1}\right)$ and $U_{2}=F_{2}\left(t_{2}\right)$ where $U_{1}$ and $U_{2}$ have uniform 
$(0,1)$ distributions, but are usually dependent if $T_{1}$ and $T_{2}$ are dependent $\left(T_{1}\right.$ and $T_{2}$ independent, implies that $U_{1}$ and $U_{2}$ are independent).

Specifying dependence between $T_{1}$ and $T_{2}$ is the same as specifying dependence between $U_{1}$ and $U_{2}$. Assuming $U_{1}$ and $U_{2}$ uniform random variables, the problem reduces to specifying a bivariate distribution between two uniforms, that is, a copula.

In this section, we introduce two special copula functions which are explored in our study: the Gumbel-Barnett and the Farlie-Gumbel-Morgenstern copulas. In all cases, $u=F_{1}\left(t_{1}\right)=$ $P\left(T_{1} \leq t_{1}\right), v=F_{2}\left(t_{2}\right)=P\left(T_{2} \leq t_{2}\right) ; C(u, v)=F\left(t_{1}, t_{2}\right)=P\left(T_{1} \leq t_{1}, T_{2} \leq t_{2}\right)$ and $\theta$ is the dependence parameter.

Also, let us assume the marginal Weibull distributions given by,

$$
u=F_{1}\left(t_{1}\right)=1-\exp \left(-\left(\frac{t_{1}}{\lambda_{1}}\right)^{p_{1}}\right)
$$

and

$$
v=F_{2}\left(t_{2}\right)=1-\exp \left(-\left(\frac{t_{2}}{\lambda_{2}}\right)^{p_{2}}\right)
$$

where $p_{1}$ and $p_{2}$ are the shape parameters of the Weibull distributions.

A copula based measure of dependence is also introduced by Ledwina(2015) given by,

$$
q(u, v)=\frac{C(u, \mathrm{v})-u \mathrm{v}}{w(u, \mathrm{v})}
$$

where $w(u, v)=[u v(1-u)(1-v)]^{0.5}$ and $B_{1}(u, v)<q(u, v)<B_{2}(u, v) ; B_{1}(u, v)=$ $w(u, v)[\max (u+v-1,0)-u v] ; B_{2}(u, v)=w(u, v)[\min (u, v)-u v]$.

\subsection{Gumbel-Barnett copula (GB copula)}

The Gumbel-Barnett copula (Gumbel,1960; Barnett, 1980) is defined by,

$$
C(u, v)=u+v-1+(1-u)(1-v) \exp \{-\theta \ln (1-u) \ln (1-v)\}
$$

for $0 \leq \theta \leq 1$. Independence corresponds to $\theta=0$.

The joint density function $f\left(t_{1}, t_{2}\right)$ for the random variables $T_{1}$ and $T_{2}$ is obtained from the second derivative of the joint distribution function with respect to $t_{1}$ and $t_{2}$, that is,

$$
\begin{gathered}
f\left(t_{1}, t_{2}\right)=f_{1}\left(t_{1}\right) f_{2}\left(t_{2}\right) \exp \left\{-\theta \log \left(1-F_{1}\left(t_{1}\right)\right) \log \left(1-F_{2}\left(t_{2}\right)\right)\right\}\{1-\theta-\theta \log (1- \\
\left.\left.F_{1}\left(t_{1}\right)\right)-\theta \log \left(1-F_{2}\left(t_{2}\right)\right)+\theta^{2} \log \left(1-F_{1}\left(t_{1}\right)\right) \log \left(1-F_{2}\left(t_{2}\right)\right)\right\}
\end{gathered}
$$

Also,

$$
F\left(t_{1}, t_{2}\right)=F_{1}\left(t_{1}\right)+F_{2}\left(t_{2}\right)-1+\left(1-F_{1}\left(t_{1}\right)\right)\left(1-F_{2}\left(t_{2}\right)\right) \exp \left\{-\theta \ln \left(1-F_{1}\left(t_{1}\right)\right) \ln \left(1-F_{2}\left(t_{2}\right)\right)\right\}
$$

From (5.7), we have, 


$$
\begin{aligned}
F_{I}\left(t_{1}, t_{2} \mid \lambda_{1}, \lambda_{2}, p_{1}, p_{2}, \theta\right)=1 & -\exp \left(-\left(\frac{t_{1}}{\lambda_{1}}\right)^{p_{1}}\right)-\exp \left(-\left(\frac{t_{2}}{\lambda_{2}}\right)^{p_{2}}\right)+\exp \left\{-\left(\frac{t_{1}}{\lambda_{1}}\right)^{p_{1}}-\right. \\
& \left.\left(\frac{t_{2}}{\lambda_{2}}\right)^{p_{2}}-\theta\left(\frac{t_{1}}{\lambda_{1}}\right)^{p_{1}}\left(\frac{t_{2}}{\lambda_{2}}\right)^{p_{2}}\right\}
\end{aligned}
$$

where $t_{1}>0$ and $t_{2}>0$.

Observe that if $\theta=0$, we have independent random variables. Also, observe that the joint bivariate survival function is given by,

$$
S\left(t_{1}, t_{2}\right)=1-F_{1}\left(t_{1}\right)-F_{2}\left(t_{2}\right)+F\left(t_{1}, t_{2}\right)
$$

where $F_{1}\left(t_{1}\right)$ and $F_{2}\left(t_{2}\right)$ are given by (5.4)and $F\left(t_{1}, t_{2}\right)$ is given by (5.8), that is,

$$
S_{I}\left(t_{1}, t_{2}\right)=\exp \left\{-\left(\frac{t_{1}}{\lambda_{1}}\right)^{p_{1}}-\left(\frac{t_{2}}{\lambda_{2}}\right)^{p_{2}}-\theta\left(\frac{t_{1}}{\lambda_{1}}\right)^{p_{1}}\left(\frac{t_{2}}{\lambda_{2}}\right)^{p_{2}}\right\}
$$

The Gumbel-Barnett copula model is denoted as "GB model".

\subsection{Farlie-Gumbel-Morgenstern copula (FGM copula)}

The Farlie-Gumbel-Morgenstern copula (Morgenstern, 1956; Nelsen, 2006) is defined by,

$$
C(u, v)=u v[1+\theta(1-u)(1-v)]
$$

for $-1 \leq \theta \leq 1$. In other words, the copula $C$ captures the dependence structure between $T_{1}$ and $T_{2}$. Independence corresponds to $\theta=0$.

The joint density function $f\left(t_{1}, t_{2}\right)$ for the random variables $T_{1}$ and $T_{2}$ is given by,

$$
f\left(t_{1}, t_{2}\right)=\mathrm{f}_{1}\left(\mathrm{t}_{1}\right) \mathrm{f}_{2}\left(\mathrm{t}_{2}\right)\left[1+\theta \mathrm{f}_{1}\left(\mathrm{t}_{1}\right) \mathrm{f}_{2}\left(\mathrm{t}_{2}\right)\left(1-2 \mathrm{~F}_{1}\left(\mathrm{t}_{1}\right)\right)\left(1-2 \mathrm{~F}_{2}\left(\mathrm{t}_{2}\right)\right)\right]
$$

From(5.4) and(5.11) we have,

$$
\begin{aligned}
F_{I I}\left(t_{1}, t_{2} \mid \lambda_{1}, \lambda_{2}, p_{1}, p_{2}, \theta\right)= & {\left[1-\exp \left(-\left(\frac{t_{1}}{\lambda_{1}}\right)^{p_{1}}\right)\right]\left[1-\exp \left(-\left(\frac{t_{2}}{\lambda_{2}}\right)^{p_{2}}\right)\right][1+\theta \exp \{} \\
& \left.\left.-\left(\frac{t_{1}}{\lambda_{1}}\right)^{p_{1}}-\left(\frac{t_{2}}{\lambda_{2}}\right)^{p_{2}}\right\}\right]
\end{aligned}
$$

The joint survival function is given by,

$$
S_{I I}\left(t_{1}, t_{2} \mid \lambda_{1}, \lambda_{2}, p_{1}, p_{2}, \theta\right)=\exp \left\{-\left(\frac{t_{1}}{\lambda_{1}}\right)^{p_{1}}-\left(\frac{t_{2}}{\lambda_{2}}\right)^{p_{2}}\right\}\left\{1+\theta\left[1-\exp \left(-\left(\frac{t_{1}}{\lambda_{1}}\right)^{p_{1}}\right)\right]\right.
$$




$$
\left.\left[1-\exp \left(-\left(\frac{t_{2}}{\lambda_{2}}\right)^{p_{2}}\right)\right]\right\}
$$

The Farlie-Gumbel-Morgenstern copula model is denoted as "FGM model".

The likelihood function for $\lambda_{1}, \lambda_{2}, p_{1}, p_{1}$, and $\theta$ assuming GB or FGM copula models with marginal Weibull distributions is obtained from(4.3).

\section{Applications}

In the estimation of the Ledwina dependence measure (3.3) considering each proposed parametrical distribution function, we use Bayesian methods. As we have posterior distributions with no closed form, we use the OpenBugs software (Spiegelhalter et al, 2003) to obtain the Bayesian estimates of the parameters (use of Markov Chain Monte Carlo or MCMC methods, see for example Gelfand and Smith, 1990 or Chib and Greenberg, 1995). For all models, 15,000 Gibbs samples were simulated from the conditional distributions for each parameter. From these generated samples, we discarded the first 5,000 samples to eliminate the effect of the initial values considering a spacing of size 100 to get the final simulated sample. Convergence of the algorithm was verified graphically.

\subsection{Application 1: Holt and Prentice (1974) data set}

In Table 1, it is presented a bivariate lifetime data set introduced by Holt and Prentice (1974) that corresponds to the survival times in days of closely $\left(T_{1}\right)$ and poorly $\left(T_{2}\right)$ matched skin grafts on the same burn patient $(n=11)$. Table 1 also presents the censoring indicators for the two lifetimes where $\delta=1$ for a complete observation and $\delta=0$ for a censored observation.

Table 1: Holt and Prentice (1974) data set

\begin{tabular}{|c|c|c|c|c|}
\hline Obs & $t_{1}$ & $t_{2}$ & $\delta_{1}$ & $\delta_{2}$ \\
\hline 1 & 37 & 29 & 1 & 1 \\
\hline 2 & 19 & 13 & 1 & 1 \\
\hline 3 & 57 & 15 & 0 & 1 \\
\hline 4 & 93 & 26 & 1 & 1 \\
\hline 5 & 16 & 11 & 1 & 1 \\
\hline 6 & 22 & 17 & 1 & 1 \\
\hline 7 & 20 & 26 & 1 & 1 \\
\hline 8 & 18 & 21 & 1 & 1 \\
\hline 9 & 63 & 43 & 1 & 1 \\
\hline 10 & 29 & 15 & 1 & 1 \\
\hline
\end{tabular}

In Table 2, it is presented the posterior summaries of interest using the three parametrical models presented in sections 4 and 5. It is assumed $\operatorname{Gamma}(1,100)$ prior distributions for the parameters $\lambda_{k}, k=1,2$ of the $\mathrm{BB}$ distribution, uniform $U(0,500)$ prior distributions for the parameters $\lambda_{k}, k=1,2$ of the Weibull marginal distributions and $U(0,5)$ prior distributions 

lifetime data in presence of censored data

for the shape parameters of the marginal Weibull distributions assuming the GB and FGM copula models. Furthermore, let us assume the prior distributions $U[0,1]$ and $U[0,0.2]$ for the parameter $\theta$ in the GB and FGM models, respectively.

In Table 3, it is presented the LY non-parametrical estimator of the joint survival function and the Bayesian estimators of the joint survival function assuming the BB, GB and FGM models using the Bayesian estimators given in Table 2. In Table 3, it is also presented the Kaplan-Meier (KM) estimates for the marginal survival functions of the lifetimes $T_{1}$ and $T_{2}$.

Table 2: Posterior means and posterior standard deviations (in parenthesis) for the parameters of the three models

\begin{tabular}{|c|c|c|c|c|c|}
\hline model & $\lambda_{1}$ & & $\lambda_{2}$ & & $\lambda_{3}$ \\
\hline BB & $0.006(0.004)$ & & $0.018(0.010)$ & & $0.025(0.010)$ \\
\hline Copula & $\lambda_{1}$ & $\lambda_{2}$ & $p_{1}$ & $p_{2}$ & $\theta$ \\
\hline GB & $63.08(23.51)$ & $27.68(4.201)$ & $1.427(0.424)$ & $2.304(0.548)$ & $0.191(0.174)$ \\
\hline FGM & $57.14(18.48)$ & $27.41(3.757)$ & $1.535(0.396)$ & $2.427(0.536)$ & $0.114(0.055)$ \\
\hline
\end{tabular}

Table 3: Estimators for the joint survival function $S\left(t_{1}, t_{2}\right)$

\begin{tabular}{|c|c|c|c|c|c|c|c|c|}
\hline pair & $t_{1}$ & $t_{2}$ & LY & KM $S\left(t_{1}\right)$ & KM $S\left(t_{2}\right)$ & BB & GB & FGM \\
\hline 1 & 16 & 11 & 0.909 & 0.909 & 0.909 & 0.544 & 0.768 & 0.779 \\
\hline 2 & 19 & 13 & 0.727 & 0.727 & 0.818 & 0.486 & 0.696 & 0.708 \\
\hline 3 & 18 & 21 & 0.454 & 0.818 & 0.454 & 0.368 & 0.490 & 0.503 \\
\hline 4 & 22 & 17 & 0.363 & 0.545 & 0.545 & 0.405 & 0.570 & 0.583 \\
\hline 5 & 20 & 26 & 0.272 & 0.636 & 0.272 & 0.298 & 0.335 & 0.343 \\
\hline 6 & 29 & 15 & 0.363 & 0.454 & 0.636 & 0.383 & 0.554 & 0.561 \\
\hline 7 & 37 & 29 & 0.181 & 0.363 & 0.181 & 0.214 & 0.186 & 0.196 \\
\hline 8 & 57 & 15 & 0.363 & 0.363 & 0.636 & 0.203 & 0.316 & 0.297 \\
\hline 9 & 60 & 40 & 0.181 & 0.363 & 0.090 & 0.099 & 0.025 & 0.029 \\
\hline 10 & 63 & 43 & 0.000 & 0.181 & 0.000 & 0.085 & 0.013 & 0.017 \\
\hline 11 & 93 & 26 & 0.000 & 0.000 & 0.272 & 0.060 & 0.055 & 0.053 \\
\hline
\end{tabular}

In Table 4, it is presented the Bayesian estimates of the Ledwina dependence measure (3.3) assuming the BB, GB and FGM and the LY non-parametric estimator for $S\left(t_{1}, t_{2}\right)$. For the estimation of the empirical Ledwina dependence measure (use of LY estimator) we used the KM product-limit estimators for the univariate lifetimes $T_{1}$ and $T_{2}$, for the estimation of the BB Ledwina dependence measure we used Bayesian estimators for the parameters of the marginal exponential distributions of the univariate lifetimes $T_{1}$ and $T_{2}$, and for the estimation of the GB and FGM Ledwina dependence measures we used Bayesian estimators for the parameters of the marginal Weibull distributions of the univariate lifetimes $T_{1}$ and $T_{2}$

Table 4: Ledwina dependence measure(3.3) for each model

\begin{tabular}{|c|l|l|l|l|l|l|}
\hline pair & $t_{1}$ & $t_{2}$ & $\begin{array}{c}\text { LED } \\
\text { EMP }\end{array}$ & $\begin{array}{c}\text { LED } \\
\text { BB }\end{array}$ & $\begin{array}{c}\text { LED } \\
\text { GB }\end{array}$ & $\begin{array}{c}\text { LED } \\
\text { FGM }\end{array}$ \\
\hline 1 & 16 & 11 & 1.0000 & 0.1604 & -0.0148 & 0.0117 \\
\hline 2 & 19 & 13 & 0.7698 & 0.1528 & -0.0217 & 0.0152 \\
\hline 3 & 18 & 21 & 0.43033 & 0.0712 & -0.0424 & 0.0203 \\
\hline
\end{tabular}


Jorge Alberto Achcar, Jose Rafael Tovar Cuevas, Fernando A. Moala

\begin{tabular}{|c|c|c|c|c|c|c|}
\hline 4 & 22 & 17 & 0.26666 & 0.1335 & -0.0360 & 0.0204 \\
\hline 5 & 20 & 26 & 0.46291 & 0.0491 & -0.0572 & 0.0216 \\
\hline 6 & 29 & 15 & 0.31053 & 0.1354 & -0.0352 & 0.0211 \\
\hline 7 & 37 & 29 & 0.62361 & 0.1057 & -0.0861 & 0.0260 \\
\hline 8 & 57 & 15 & 0.57143 & -0.0190 & -0.0217 & 0.0222 \\
\hline 9 & 60 & 40 & 1.07571 & 0.0716 & -0.0685 & 0.0148 \\
\hline 10 & 63 & 43 & $*$ & 0.0683 & -0.0503 & 0.0115 \\
\hline 11 & 93 & 26 & $*$ & -0.0665 & -0.0975 & 0.0183 \\
\hline
\end{tabular}

In Figure 1 it is presented the plots of the Ledwina dependence measures considering each fitted model. From these plots, it is possible to see that the BB bivariate exponential distribution is better in comparison with the other copula models, since the plot of the estimated Ledwina BB measure is more close to the empirical Ledwina measure using the LY non-parametric estimator, although the data set has a very small sample size. The two copula models (GB and FGM models) give similar fit for the data set.

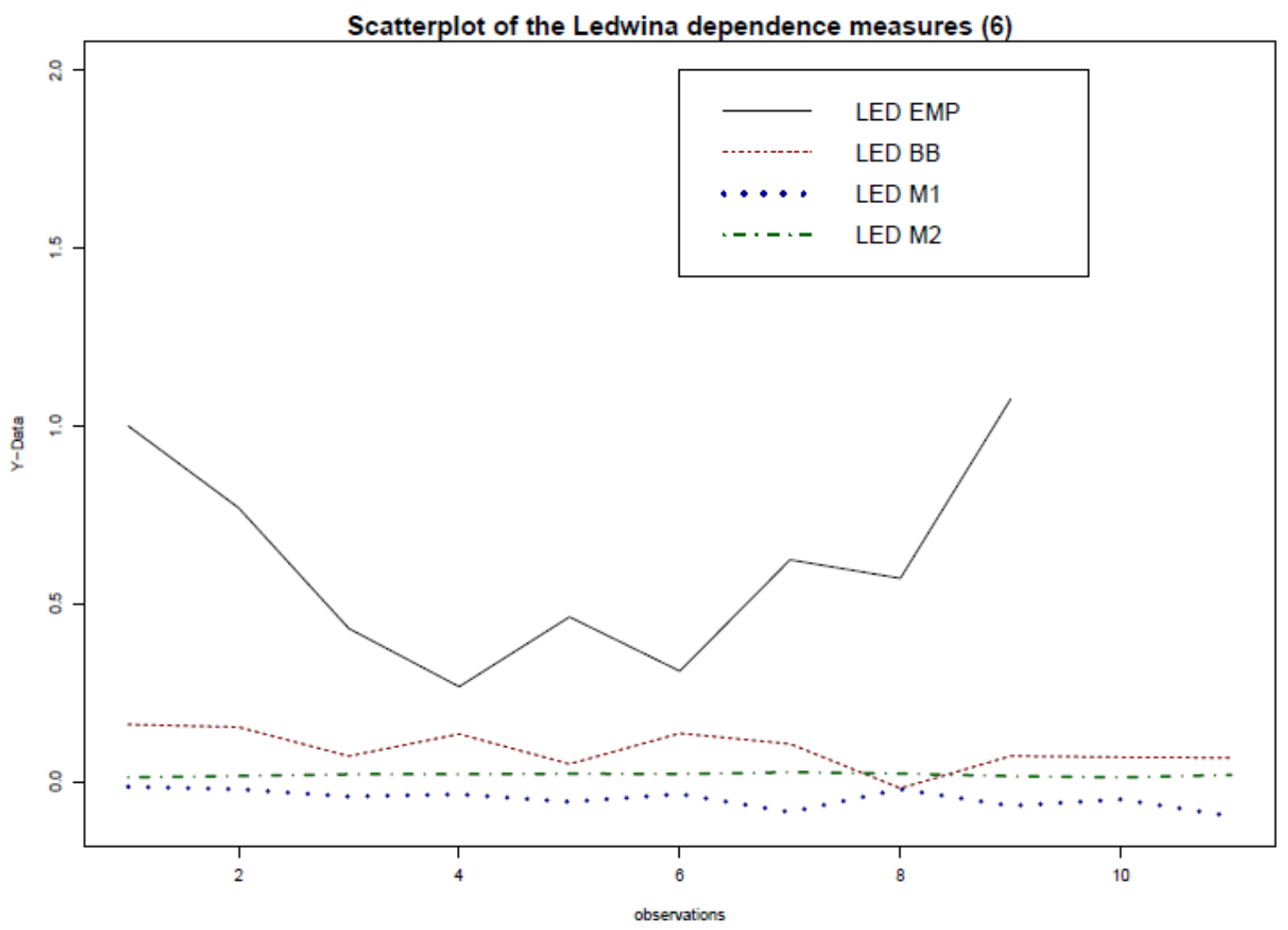

Figure 1: Plots of the Ledwina dependence measures for each fitted model

\subsection{Application 2: bone marrow transplant data set}

Bone marrow transplants are a standard treatment for acute leukemia. Prognosis for recovery may depend on risk factors known at the time of transplantation, such as patient and/or donor age and sex, the stage of initial disease, the time from prognosis to transplantation among many other factors. The final prognosis may change as the patient post- 

lifetime data in presence of censored data

transplantation history develops with occurrence of events at random times during the recovery process, such as development of accurate or chronic graft-versus-host disease (GVHD), return of the platet count to normal levels, or development of infections. In this study, 137 patients with leukemia received a combination of $16 \mathrm{mg} / \mathrm{kg}$ of oral Busulfan (BU) and $120 \mathrm{mg} / \mathrm{kg}$ of intravenous cyclophosphamide $(\mathrm{Cy})$ and were treated at one of four hospitals: 76 at Ohio State University hospitals (OSU) in Columbus; 21 at Hahnemann University (HU) in Philadelphia; 23 at St. Vincent's Hospital (SVH) in Sidney, Australia and 17 at Alfred Hospital (AH) in Melbourne, Australia (data set introduced by Klein and Moeschberger, 1997, page 464).

In the analysis considered in this section, it is assumed as lifetimes, the time (in days) to accurate graft-versus-host disease (TA) with 111 censored observa- tions and 26 not-censored observations and the time (in days) to chronic graft- versus-host disease (TC) with 76 censored observations and 61 not-censored observations, that is, we have a great number of censored observations as it is common in medical studies. In this situation, usually it is very difficult to decide by an appropriate parametrical model to be fitted by the data set.

In Table 5, it is presented the posterior summaries of interest using the three parametrical models presented in sections 4 and 5 assuming the same prior distributions given in application 1 .

Table 5: Posterior means and posterior standard deviation (in parenthesis) for the parameters of the three models

\begin{tabular}{|c|c|c|c|c|c|}
\hline model & $\lambda_{1}$ & & $\lambda_{2}$ & & $\lambda_{3}$ \\
\hline BB & $0.0003(0.0000)$ & & $0.0009(0.0001)$ & & $0.0000(0.0000)$ \\
\hline Copula & $\lambda_{1}$ & $\lambda_{2}$ & $p_{1}$ & $p_{2}$ & $\theta$ \\
\hline GB & $260700(2827000)$ & $1277(267.20)$ & $0.326(0.058)$ & $0.697(0.069)$ & $0.094(0.086)$ \\
\hline FGM & $258700(1089000)$ & $1232(249.30)$ & $0.327(0.061)$ & $0.710(0.071)$ & $0.295(0.128)$ \\
\hline
\end{tabular}

In Table 6 (see at the end of this paper) it is presented the LY non-parametrical estimator of the joint survival function and the Bayesian estimators for the parameters of the joint survival function assuming the BB, GB and FGM models and using the Bayesian estimators given in Table 5.

In Table 7 (placed at the end of this paper), it is presented the Bayesian estimators of the Ledwina dependence measure (3.3) assuming the BB, GB and FGM and the LY nonparametric estimator of $S\left(t_{1}, t_{2}\right)$. For the estimation of the empirical Ledwina dependence measure (use of LY estimator) we have used the KM product-limit estimators for the univariate lifetimes $T_{1}$ and $T_{2}$; for the estimation of the BB Ledwina dependence measure we used Bayesian estimators for the parameters of the marginal exponential distributions for the univariate lifetimes $T_{1}$ and $T_{2}$ and for the estimation of the GB and FGM Ledwina dependence measures we used Bayesian estimators for the parameters of the marginal Weibull distributions of the univariate lifetimes $T_{1}$ and $T_{2}$.

In Figure 2 it is presented the plots of the Ledwina dependence measures considering each fitted model. From these plots, it is possible to see that the BB bivariate exponential distribution is better fitted by the data in comparison with the other copula models, since the plot of the estimated Ledwina BB measure is more close to the empirical Ledwina measure using the LY non-parametric estimator. The good fit of the BB bivariate exponential distribution is also observed in Figure 3. 
It is important to point out that other bivariate lifetime distributions could be assumed to this data set with possible better fit. In this way, we have a powerful graphical approach to find appropriate bivariate distributions for bivariate lifetime data in presence of censoring.

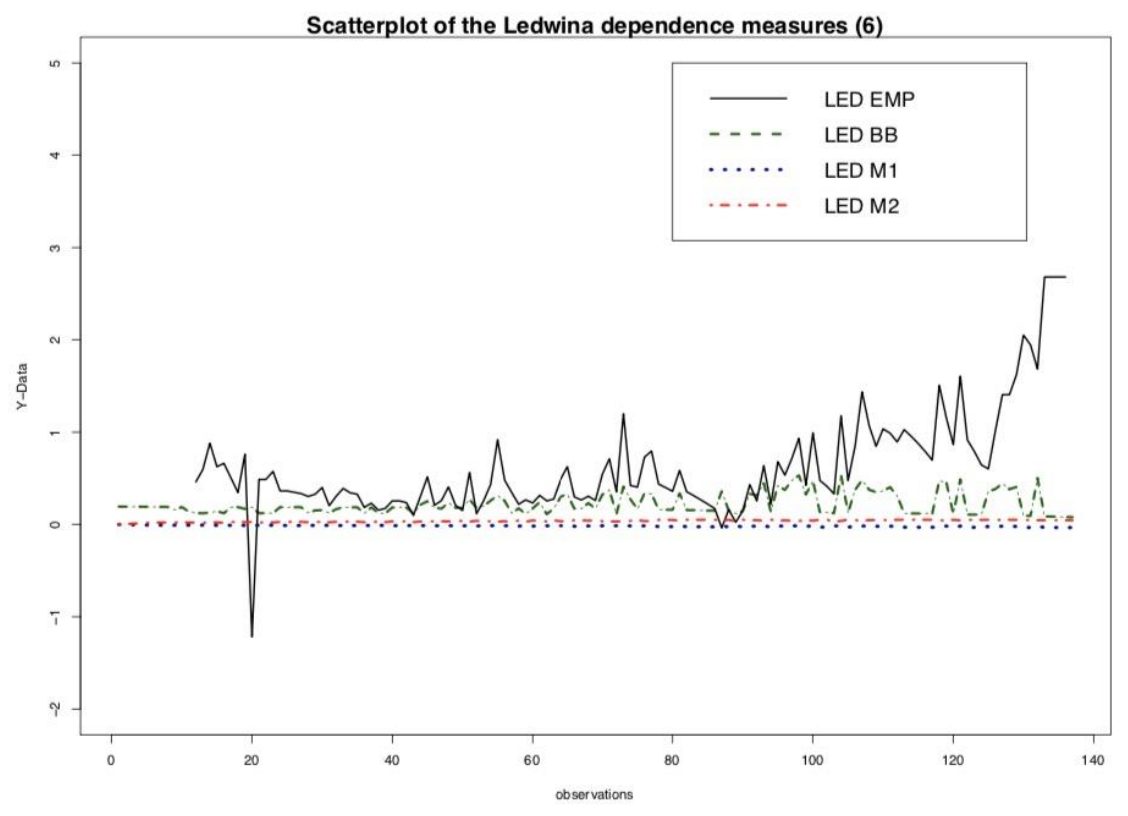

Figure 2: Plots of the Ledwina dependence measures for each fitted model

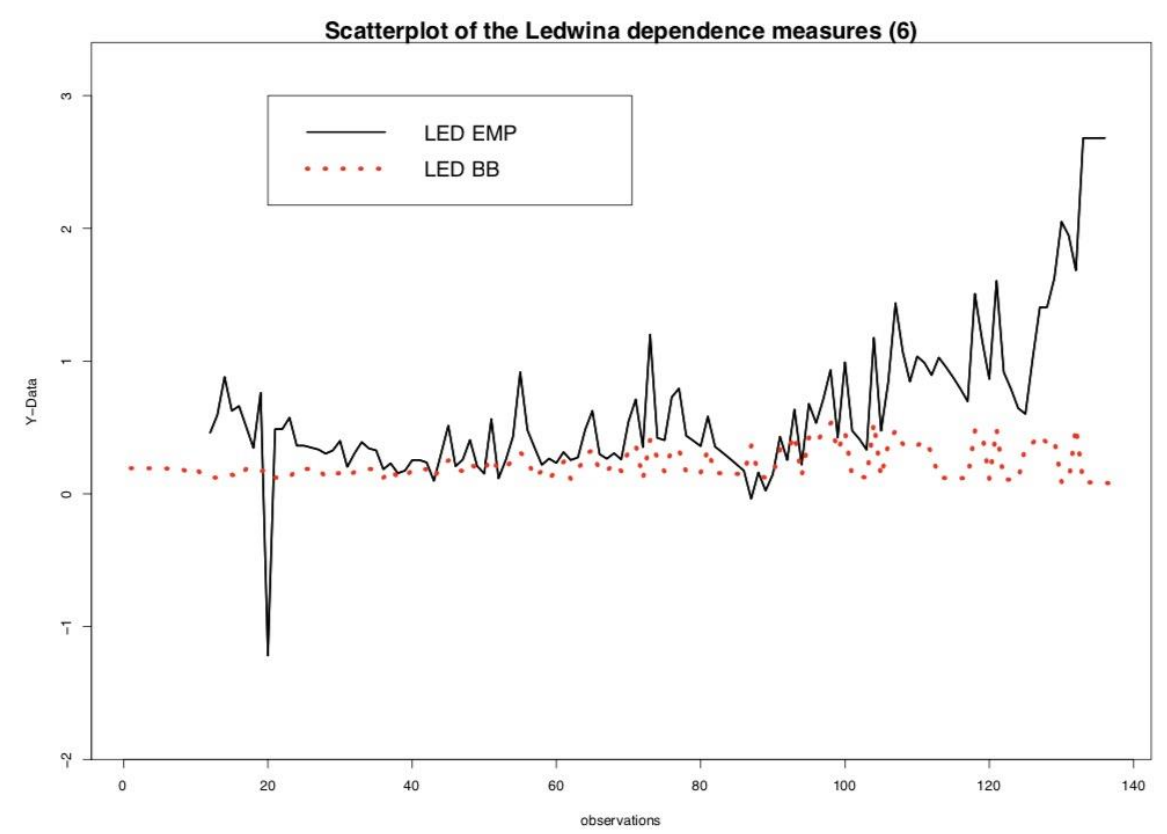

Figure 3: Plots of the Ledwina dependence measures considering the empirical LY nonparametric estimator and the BB bivariate lifetime distribution

\subsection{Application 3: UEFA Champion League data}

In this application, let us consider a data set obtained from Meintanis (2007) and introduced in Table 8. The data represent 37 football matches from the UEFA Champion's League 2004- 

lifetime data in presence of censored data

2005 and 2005-2006 seasons where at least one goal scored by the home team and at least one goal scored directly from a penalty kick, foul kick or any other direct kick by any team have been considered. In this way $T_{1}$ represents the time in minutes of the first kick goal scored by any team and $T_{2}$ represents the first goal of any type scored by the home team. In this case, all possibilities are open, for example, $T_{1}<T_{2}, T_{1}>T_{2}$ or $T_{1}=T_{2}=Y$.

Let us assume marginal exponential distributions with distribution functions given by,

$$
F_{i}\left(t_{i}\right)=P\left\{T_{i} \leq t_{i}\right\}=1-\exp \left(-\lambda_{i} t_{i}\right), i=1,2
$$

and gamma prior distributions with hyperparameters $a=0.1$ and $b=0.1$ for the parameters $\lambda_{1}$ and $\lambda_{2}$ assuming the GB and FGM copula models (denoted as M1 and M2 models).

Table 8: UEFA Champion's League data (2004-2006)

\begin{tabular}{|c|c|c|c|c|c|}
\hline match & $T_{1}$ & $T_{2}$ & match & $T_{1}$ & $T_{2}$ \\
\hline Lyon $\times$ Real Madrid & 26 & 20 & Internazionale $\times$ Bremen & 34 & 34 \\
\hline Milan $\times$ Fenerbahce & 63 & 18 & Real Madrid $\times$ Roma & 53 & 39 \\
\hline Chelsea $\times$ Anderlecht & 19 & 19 & Man. United $\times$ Fenerbahce & 54 & 7 \\
\hline Club BruggexJuventus & 66 & 85 & Bayern $\times$ Ajax & 51 & 28 \\
\hline Fenerbahce $\times$ PSV & 40 & 40 & Moscow $\times$ PSG & 76 & 64 \\
\hline Internazionale $\times$ Rangers & 49 & 49 & Barcelona $\times$ Shakhtar & 64 & 15 \\
\hline Panathinaikis $\times$ Bremen & 8 & 8 & Leverkusen $\times$ Roma & 26 & 48 \\
\hline Ajax-Arsenal & 69 & 71 & Arsenal×Panathinaikos & 16 & 16 \\
\hline Man. United $\times$ Benfica & 39 & 39 & Dynamo Kyiv×Real Madrid & 44 & 13 \\
\hline Real Madrid $\times$ Rosenborg & 82 & 48 & Man. United $\times$ Sparta & 25 & 14 \\
\hline Vilarreal×Benfica & 72 & 72 & Bayern $\times$ M. TelAviv & 55 & 11 \\
\hline Juventus $\times$ Bayern & 66 & 62 & Bremen $\times$ Internazionale & 49 & 49 \\
\hline Club BruggexRapid & 25 & 9 & Anderlecht $\times$ Valencia & 24 & 24 \\
\hline Olympiacos $\times$ Lyon & 41 & 3 & Panathinaikos $\times \mathrm{PSV}$ & 44 & 30 \\
\hline InternazionalexPorto & 16 & 75 & Arsenal×Rosenborg & 42 & 3 \\
\hline Schalke $\times$ PSV & 18 & 18 & Liverpool×Olympiacos & 27 & 47 \\
\hline Barcelona $\times$ Bremen & 22 & 14 & M. TelAviv $\times$ Juventus & 28 & 28 \\
\hline Milan $\times$ Schalke & 42 & 42 & Bremen $\times$ Panathinaikos & 2 & 2 \\
\hline Rapid $\times$ Juventus & 36 & 52 & & & \\
\hline
\end{tabular}

Table 9 presents the posterior summaries of interest using the three parametrical models presented in sections 4 and 5.

Table 9: Posterior means and posterior standard deviation (in parenthesis) for the parameters of the three models

\begin{tabular}{|c|c|c|c|}
\hline model & $\lambda_{1}$ & $\lambda_{2}$ & $\lambda_{3}$ \\
\hline BB & $0.0001(0.0004)$ & $0.0007(0.0015)$ & $0.0444(0.0053)$ \\
\hline Copula & $\lambda_{1}$ & $\lambda_{2}$ & $\theta$ \\
\hline GB & $0.0244(0.0040)$ & $0.0297(0.0049)$ & $0.1695(0.1575)$ \\
\hline FGM & $0.0254(0.0040)$ & $0.0323(0.0053)$ & $0.6748(0.2850)$ \\
\hline
\end{tabular}


Jorge Alberto Achcar, Jose Rafael Tovar Cuevas, Fernando A. Moala

In Table 10, it is presented the KM non-parametrical estimator of the joint survival function and the Bayesian estimators of the joint survival function assuming the GB and FGM models using the Bayesian estimators given in Table 9.

Table 10: Estimators for the joint survival function $\mathrm{S}\left(t_{1}, t_{2}\right)$

\begin{tabular}{|c|c|c|c|c|c|c|}
\hline pair & $t_{1}$ & $t_{2}$ & KM & BB & GB & FGM \\
\hline 1 & 26 & 20 & 0.4864 & 0.1205 & 0.2838 & 0.3096 \\
\hline 2 & 63 & 18 & 0.1621 & 0.1073 & 0.1157 & 0.1378 \\
\hline 3 & 19 & 19 & 0.5675 & 0.0713 & 0.3517 & 0.3702 \\
\hline 4 & 66 & 85 & 0.000002 & 0.0243 & 0.0090 & 0.0174 \\
\hline 5 & 40 & 40 & 0.2432 & 0.0275 & 0.1001 & 0.1279 \\
\hline 6 & 49 & 49 & 0.1351 & 0.0183 & 0.0565 & 0.0797 \\
\hline 7 & 8 & 8 & 0.8648 & 0.1173 & 0.6510 & 0.6455 \\
\hline 8 & 69 & 71 & 0.0270 & 0.0407 & 0.0137 & 0.0253 \\
\hline 9 & 39 & 39 & 0.2702 & 0.0288 & 0.1065 & 0.1347 \\
\hline 10 & 82 & 48 & 0.0008 & 0.0352 & 0.0220 & 0.0376 \\
\hline 11 & 72 & 72 & 0.00009 & 0.0065 & 0.0120 & 0.0228 \\
\hline 12 & 66 & 62 & 0.0810 & 0.0159 & 0.0211 & 0.0360 \\
\hline 13 & 25 & 9 & 0.5405 & 0.2464 & 0.3007 & 0.3254 \\
\hline 14 & 41 & 3 & 0.4594 & 0.2491 & 0.3406 & 0.3315 \\
\hline 15 & 16 & 75 & 0.0270 & 0.0486 & 0.0676 & 0.0689 \\
\hline 16 & 18 & 18 & 0.6216 & 0.0746 & 0.3724 & 0.3891 \\
\hline 17 & 22 & 14 & 0.6486 & 0.1719 & 0.3809 & 0.3991 \\
\hline 18 & 42 & 42 & 0.2162 & 0.0251 & 0.0883 & 0.1152 \\
\hline 19 & 36 & 52 & 0.1351 & 0.1064 & 0.0753 & 0.0968 \\
\hline 20 & 34 & 34 & 0.3513 & 0.0361 & 0.1449 & 0.1741 \\
\hline 21 & 53 & 39 & 0.1621 & 0.0625 & 0.0714 & 0.0981 \\
\hline 22 & 54 & 7 & 0.2433 & 0.1680 & 0.2159 & 0.2212 \\
\hline 23 & 51 & 28 & 0.1891 & 0.1020 & 0.1112 & 0.1408 \\
\hline 24 & 76 & 64 & 0.000005 & 0.0197 & 0.0143 & 0.0266 \\
\hline 25 & 64 & 15 & 0.1621 & 0.1110 & 0.1258 & 0.1447 \\
\hline 26 & 26 & 48 & 0.2162 & 0.1327 & 0.1157 & 0.1347 \\
\hline 27 & 16 & 16 & 0.6756 & 0.0816 & 0.4173 & 0.4300 \\
\hline 28 & 44 & 13 & 0.3243 & 0.1812 & 0.2249 & 0.2461 \\
\hline 29 & 25 & 14 & 0.5945 & 0.1861 & 0.3528 & 0.3731 \\
\hline 30 & 55 & 11 & 0.2162 & 0.1511 & 0.1827 & 0.1978 \\
\hline 31 & 49 & 49 & 0.1351 & 0.0183 & 0.0565 & 0.0797 \\
\hline 32 & 24 & 24 & 0.5135 & 0.0568 & 0.2633 & 0.2885 \\
\hline 33 & 44 & 30 & 0.2433 & 0.0940 & 0.1257 & 0.1565 \\
\hline 34 & 42 & 3 & 0.4324 & 0.2438 & 0.3325 & 0.3234 \\
\hline 35 & 27 & 47 & 0.2432 & 0.1371 & 0.1159 & 0.1362 \\
\hline 36 & 28 & 28 & 0.4054 & 0.0474 & 0.2079 & 0.2361 \\
\hline 37 & 2 & 2 & 0.9729 & 0.1539 & 0.8996 & 0.8919 \\
\hline & & & & & & \\
\hline
\end{tabular}

In Table 11, it is presented the Bayesian estimates of the Ledwina dependence measure (3.3) assuming the BB, GB and FGM and the LY non-parametric estimator for $S\left(t_{1}, t_{2}\right)$ : for the estimation of the empirical Ledwina dependence measure (use of LY estimator) we used the KM 

lifetime data in presence of censored data

product-limit estimators for the univariate lifetimes $T_{1}$ and $T_{2}$ for the estimation of the BB Ledwina dependence measure we used Bayesian estimators for the parameters of the marginal exponential dis- tributions of the univariate lifetimes $T_{1}$ and $T_{2}$, and for the estimation of the GB and FGM Ledwina dependence measures we used Bayesian estimators for the parameters of the marginal Weibull distributions of the univariate lifetimes $T_{1}$ and $T_{2}$.

Table 11: Ledwina dependence measure (3.3) for each model

\begin{tabular}{|c|c|c|c|c|c|c|}
\hline pair & $T_{1}$ & $T_{2}$ & LED EMP & LED BB & LED GB & FED \\
\hline 1 & 26 & 20 & 0.4441 & -0.6780 & $-0,0746$ & 0.1669 \\
\hline 2 & 63 & 18 & 0.2109 & 0.0400 & $-0,0846$ & 0.1330 \\
\hline 3 & 19 & 19 & 0.3834 & -1.2631 & $-0,0660$ & 0.1621 \\
\hline 4 & 66 & 85 & 0.0090 & 0.2564 & $-0,0814$ & 0.0627 \\
\hline 5 & 40 & 40 & 0.2632 & -0.3003 & $-0,0964$ & 0.1426 \\
\hline 6 & 49 & 49 & 0.4023 & -0.1719 & $-0,0987$ & 0.1212 \\
\hline 7 & 8 & 8 & 0.6047 & -4.3109 & $-0,0326$ & 0.1091 \\
\hline 8 & 69 & 71 & 0.2745 & 0.3470 & $-0,0882$ & 0.0750 \\
\hline 9 & 39 & 39 & 0.2720 & -0.3194 & $-0,0958$ & 0.1448 \\
\hline 10 & 82 & 48 & 0.0055 & 0.1764 & $-0,0926$ & 0.0893 \\
\hline 11 & 72 & 72 & -0.0571 & -0.0260 & $-0,0866$ & 0.0717 \\
\hline 12 & 66 & 62 & 0.6261 & 0.0007 & $-0,0932$ & 0.0879 \\
\hline 13 & 25 & 9 & 0.4891 & -0.9140 & $-0,0725$ & 0.1664 \\
\hline 14 & 41 & 3 & 0.0910 & -0.7171 & $-0,0381$ & 0.0929 \\
\hline 15 & 16 & 75 & 0.0580 & 0.0815 & $-0,0733$ & 0.0885 \\
\hline 16 & 18 & 18 & 0.3714 & -1.3758 & $-0,0635$ & 0.1602 \\
\hline 17 & 22 & 14 & 0.2086 & -0.9808 & $-0,0623$ & 0.1595 \\
\hline 18 & 42 & 42 & 0.3275 & -0.2655 & $-0,0974$ & 0.1380 \\
\hline 19 & 36 & 52 & 0.2360 & 0.2647 & $-0,0954$ & 0.1265 \\
\hline 20 & 34 & 34 & 0.3874 & -0.4361 & $-0,0917$ & 0.1550 \\
\hline 21 & 53 & 39 & 0.2781 & 0.0080 & $-0,0987$ & 0.1314 \\
\hline 22 & 54 & 7 & 0.1973 & -0.1940 & $-0,0588$ & 0.1171 \\
\hline 23 & 51 & 28 & 0.1950 & 0.0083 & $-0,0941$ & 0.1458 \\
\hline 24 & 76 & 64 & -0.0580 & 0.1022 & $-0,0891$ & 0.0771 \\
\hline 25 & 64 & 15 & 0.2677 & 0.0164 & $-0,0799$ & 0.1292 \\
\hline 26 & 26 & 48 & 0.2582 & 0.2128 & $-0,0889$ & 0.1356 \\
\hline 27 & 16 & 16 & 0.3448 & -1.6488 & $-0,0582$ & 0.1550 \\
\hline 28 & 44 & 13 & 0.0922 & -0.1781 & $-0,0733$ & 0.1490 \\
\hline 29 & 25 & 14 & 0.2223 & -0.7680 & $-0,0650$ & 0.1609 \\
\hline 30 & 55 & 11 & 0.2310 & -0.0899 & $-0,0709$ & 0.1322 \\
\hline 31 & 49 & 49 & 0.4023 & -0.1719 & $-0,0987$ & 0.1212 \\
\hline 32 & 24 & 24 & 0.4379 & -0.8548 & $-0,0769$ & 0.1659 \\
\hline 33 & 44 & 30 & 0.2871 & -0.0961 & $-0,0939$ & 0.1516 \\
\hline 34 & 42 & 3 & 0.2592 & -0.6856 & $-0,0383$ & 0.0923 \\
\hline 35 & 27 & 47 & 0.2309 & 0.2248 & $-0,0898$ & 0.1373 \\
\hline 36 & 28 & 28 & 0.4248 & -0.6452 & $-0,0839$ & 0.1637 \\
\hline 37 & 2 & 2 & 1.0000 & -22.0708 & $-0,0088$ & 0.0353 \\
\hline & & & & & & \\
\hline
\end{tabular}


Figure 4 shows the plots of the Ledwina dependence measures considering each fitted model. From these plots, it is observed better fit of the M2 (FGM) model given that, plot of estimated Ledwina model very close to the plot of the empirical Ledwina measure.

\subsection{Application 4: Recurrence Times of Infection for Kidney Patients}

In this application, let us consider a survival data set introduced by McGilchrist and Aisbett (1991) related to kidney infection where the recurrence of infection of 38 kidney patients,

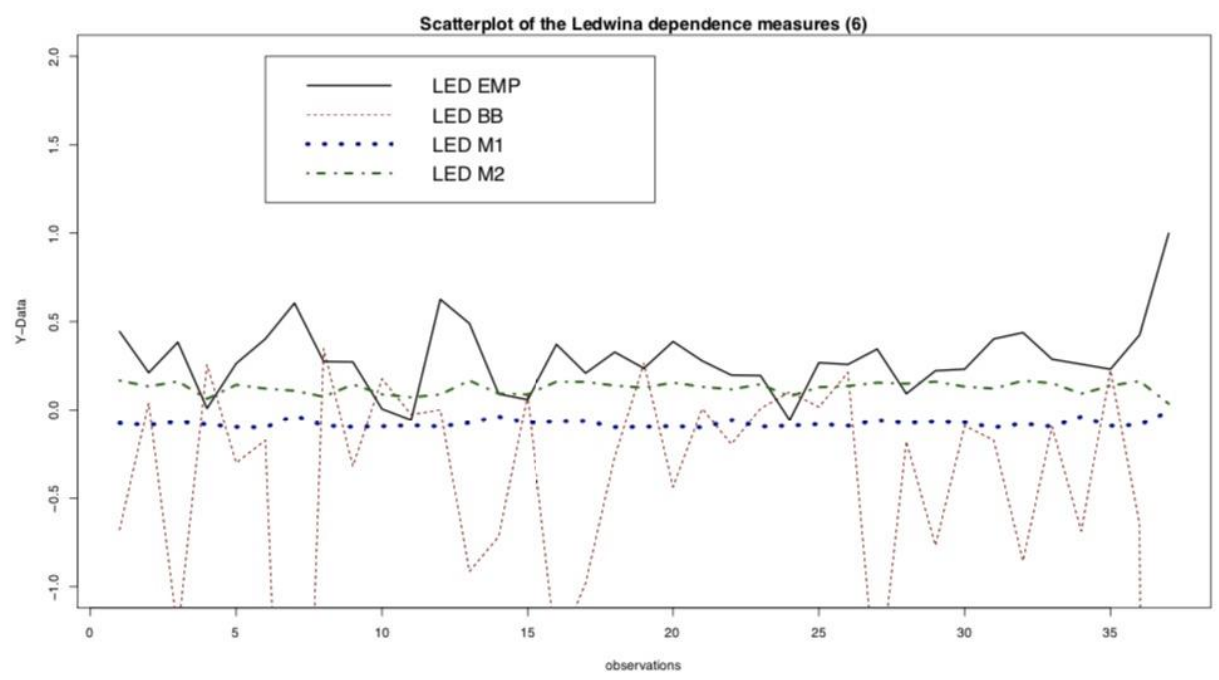

Figure 4: Plots of the Ledwina dependence measures for each fitted model

using portable dialysis machines, are recorded. Infections may occur at the location of insertion of the catheter. The time recorded, called infection time, is either the survival time (in days) of the patient until an infection occurred and the catheter had to be removed, or the censored time, where the catheter was removed by others reasons. The catheter is reinserted after some time and the second infection time is again observed or censored (data set in Table 12). To analyse this application, let us assume the three bivariate distributions obtained from a BB distribution, a GB and a FGM copula function models under marginal Weibull distributions, introduced in sections 3 and 5. As a first analysis of the recurrence times of infections, let us assume the BB exponential distribution with density (4.1). For a Bayesian analysis of the $\mathrm{BB}$ distribution, it is assumed gamma prior distributions (4.9) for the parameters $\lambda_{1}, \lambda_{2}$ and $\lambda_{3}$, with hyperparameters values $\mathrm{a}_{1}=\mathrm{a}_{2}=\mathrm{a}_{3}=1$ and $\mathrm{a}_{1}=\mathrm{a}_{2}=\mathrm{a}_{3}=$ 100.

Table 12: Recurrence times of infections in 38 kidney patients

\begin{tabular}{|c|c|c|c|c|}
\hline patient & $t_{1}$ & $t_{2}$ & $\delta_{1}$ & $\delta_{2}$ \\
\hline 1 & 8 & 16 & 1 & 1 \\
\hline 2 & 23 & 13 & 1 & 0 \\
\hline 3 & 22 & 28 & 1 & 1 \\
\hline 4 & 447 & 318 & 1 & 1 \\
\hline 5 & 30 & 12 & 1 & 1 \\
\hline
\end{tabular}



lifetime data in presence of censored data

\begin{tabular}{|c|c|c|c|c|}
\hline 6 & 24 & 245 & 1 & 1 \\
\hline 7 & 7 & 9 & 1 & 1 \\
\hline 8 & 511 & 30 & 1 & 1 \\
\hline 9 & 53 & 196 & 1 & 1 \\
\hline 10 & 15 & 154 & 1 & 1 \\
\hline 11 & 7 & 333 & 1 & 1 \\
\hline 12 & 141 & 8 & 1 & 0 \\
\hline 13 & 96 & 38 & 1 & 1 \\
\hline 14 & 149 & 70 & 0 & 0 \\
\hline 15 & 536 & 25 & 1 & 0 \\
\hline 16 & 17 & 4 & 1 & 0 \\
\hline 17 & 185 & 117 & 1 & 1 \\
\hline 18 & 292 & 114 & 1 & 1 \\
\hline 19 & 22 & 159 & 0 & 0 \\
\hline 20 & 15 & 108 & 1 & 0 \\
\hline 21 & 152 & 562 & 1 & 1 \\
\hline 22 & 402 & 24 & 1 & 0 \\
\hline 23 & 13 & 66 & 1 & 1 \\
\hline 24 & 39 & 46 & 1 & 0 \\
\hline 25 & 12 & 40 & 1 & 1 \\
\hline 26 & 113 & 201 & 0 & 1 \\
\hline 27 & 132 & 156 & 1 & 1 \\
\hline 28 & 34 & 30 & 1 & 1 \\
\hline 29 & 2 & 25 & 1 & 1 \\
\hline 30 & 130 & 26 & 1 & 1 \\
\hline 31 & 27 & 58 & 1 & 1 \\
\hline 32 & 5 & 43 & 0 & 1 \\
\hline 33 & 152 & 30 & 1 & 1 \\
\hline 34 & 190 & 5 & 1 & 0 \\
\hline 35 & 119 & 8 & 1 & 1 \\
\hline 36 & 54 & 16 & 0 & 0 \\
\hline 37 & 6 & 78 & 0 & 1 \\
\hline 38 & 63 & 8 & 1 & 0 \\
\hline
\end{tabular}

For a Bayesian analysis of bivariate Weibull distribution under GB and FGM copula functions with joint distribution functions given by (5.8) and (5.13), respectively, let us assume uniform prior distributions with hyperparameter values $\mathrm{a}_{1}=\mathrm{a}_{2}=0 ; \mathrm{b}_{1}=\mathrm{b}_{2}=500$, $\mathrm{c}_{1}=\mathrm{c}_{2}=0 ; \mathrm{d}_{1}=\mathrm{d}_{2}=2 ; e=0$ and $f=0.2$ for $\lambda_{1}, \lambda_{2}, p_{1}, p_{2}$ and $\theta$. To simulate samples of the joint posterior distribution for the parameters of the three models, we have used the WinBUGS software which requires only the specification of the joint distribution for the data and the prior distributions for the parameters of the model. In the simulation procedure, we discarded the first 5000 simulated Gibbs samples ("burnin-samples") to eliminate the effect of the initial values for the parameters of the model. Choosing every 20th simulated Gibbs sample, we obtained a final sample of size 5000 to get the posterior summaries of interest. Convergence of the Gibbs sampling algorithm was monitored using standard existing methods as time series for the simulated samples. 
Table 13 presents the posterior means and the posterior standard-deviations for the parameters for the three parametrical models presented in sections 3 and 5 .

Table 13: Posterior means and posterior standard deviation (in parenthesis) for the parameters of the three models

\begin{tabular}{|c|c|c|c|c|c|}
\hline model & $\lambda_{1}$ & & $\lambda_{2}$ & & $\lambda_{3}$ \\
\hline BB & $0.006(0.002)$ & & $0.005(0.002)$ & & $0.003(0.002)$ \\
\hline Copula & $\lambda_{1}$ & $\mathrm{p}_{1}$ & $\lambda_{2}$ & $p_{2}$ & $\theta$ \\
\hline GB & $135.3(32.96)$ & $0.805(0.110)$ & $144.6(31.66)$ & $0.999(0.141)$ & $0.077(0.055)$ \\
\hline FGM & $136.1(32.89)$ & $0.827(0.011)$ & $143.2(30.22)$ & $0.997(0.014)$ & $0.099(0.057)$ \\
\hline
\end{tabular}

In Table 14, it is presented the LY non-parametrical estimator of the joint survival function and the Bayesian estimators of the joint survival function assuming the BB, GB and FGM models using the Bayesian estimators given in Table 13. In Table 14, it is also presented the Kaplan-Meier (KM) estimates for the marginal survival functions of the lifetimes $T_{1}$ and $T_{2}$.

In Table 15, it is presented the Bayesian estimates of the Ledwina dependence measure (3.3) assuming the $\mathrm{BB}, \mathrm{GB}$ and FGM and the LY non-parametric estimator for $\mathrm{S}\left(t_{1} t_{2}\right)$. For the estimation of the empirical Ledwina dependence measure (use of LY estimator) we used the KM product-limit estimators for the univariate lifetimes $T_{1}$ and $T_{2}$, for the estimation of the BB Ledwina dependence measure we used Bayesian estimators for the parameters of the marginal exponential dis- tributions of the univariate lifetimes $T_{1}$ and $T_{2}$, and for the estimation of the GB and FGM Ledwina dependence measures we used Bayesian estimators for the parameters of the marginal Weibull distributions of the univariate lifetimes $T_{1}$ and $T_{2}$.

Table 14: Estimators for the joint survival function $S\left(t_{1} t_{2}\right)$

\begin{tabular}{|c|c|c|c|c|c|c|}
\hline patient & $\mathrm{t} 1$ & $\mathrm{t} 2$ & LY & BB & GB & FGM \\
\hline 1 & 8 & 16 & 0.632 & 0.8464 & 0.8053 & 0.8127 \\
\hline 2 & 23 & 13 & 0.530 & 0.4038 & 0.7162 & 0.7266 \\
\hline 3 & 22 & 28 & 0.418 & 0.7054 & 0.6493 & 0.6607 \\
\hline 4 & 447 & 318 & 0.000 & 0.0025 & 0.0053 & 0.0081 \\
\hline 5 & 30 & 12 & 0.474 & 0.3901 & 0.6810 & 0.6917 \\
\hline 6 & 24 & 245 & 0.107 & 0.1342 & 0.1400 & 0.1453 \\
\hline 7 & 7 & 9 & 0.737 & 0.8944 & 0.8551 & 0.8618 \\
\hline 8 & 511 & 30 & 0.000 & 0.0078 & 0.0418 & 0.0415 \\
\hline 9 & 53 & 196 & 0.143 & 0.1657 & 0.1541 & 0.1655 \\
\hline 10 & 15 & 154 & 0.280 & 0.2910 & 0.2870 & 0.2932 \\
\hline 11 & 7 & 333 & 0.054 & 0.0726 & 0.0915 & 0.0909 \\
\hline 12 & 141 & 8 & 0.339 & 0.1848 & 0.3344 & 0.3387 \\
\hline 13 & 96 & 38 & 0.237 & 0.2089 & 0.3533 & 0.3666 \\
\hline 14 & 149 & 70 & 0.160 & 0.1153 & 0.2001 & 0.2138 \\
\hline 15 & 536 & 25 & 0.000 & 0.0064 & 0.0389 & 0.0380 \\
\hline 16 & 17 & 4 & 0.731 & 0.4498 & 0.8048 & 0.8133 \\
\hline 17 & 185 & 117 & 0.043 & 0.0645 & 0.1133 & 0.1265 \\
\hline 18 & 292 & 114 & 0.054 & 0.0296 & 0.0632 & 0.0719 \\
\hline 19 & 22 & 159 & 0.216 & 0.2679 & 0.2593 & 0.2676 \\
\hline
\end{tabular}



lifetime data in presence of censored data

\begin{tabular}{|c|c|c|c|c|c|c|}
\hline 20 & 15 & 108 & 0.320 & 0.4140 & 0.3950 & 0.4032 \\
\hline 21 & 152 & 562 & 0.000 & 0.0048 & 0.0052 & 0.0071 \\
\hline 22 & 402 & 24 & 0.201 & 0.0203 & 0.0740 & 0.0739 \\
\hline 23 & 13 & 66 & 0.388 & 0.5731 & 0.5396 & 0.5486 \\
\hline 24 & 39 & 46 & 0.240 & 0.5522 & 0.4976 & 0.5118 \\
\hline 25 & 12 & 40 & 0.418 & 0.6958 & 0.6535 & 0.6627 \\
\hline 26 & 113 & 201 & 0.107 & 0.1090 & 0.0961 & 0.1089 \\
\hline 27 & 132 & 156 & 0.080 & 0.1334 & 0.1177 & 0.1321 \\
\hline 28 & 34 & 30 & 0.279 & 0.3298 & 0.5799 & 0.5928 \\
\hline 29 & 2 & 25 & 0.697 & 0.8291 & 0.8106 & 0.8142 \\
\hline 30 & 130 & 26 & 0.284 & 0.1783 & 0.3120 & 0.3214 \\
\hline 31 & 27 & 58 & 0.249 & 0.5521 & 0.5034 & 0.5164 \\
\hline 32 & 5 & 43 & 0.491 & 0.7145 & 0.6888 & 0.6944 \\
\hline 33 & 152 & 30 & 0.120 & 0.1476 & 0.2653 & 0.2742 \\
\hline 34 & 190 & 5 & 0.238 & 0.1289 & 0.2582 & 0.2591 \\
\hline 35 & 119 & 8 & 0.391 & 0.2179 & 0.3819 & 0.3875 \\
\hline 36 & 54 & 16 & 0.404 & 0.3248 & 0.5519 & 0.5633 \\
\hline 37 & 6 & 78 & 0.406 & 0.5496 & 0.5339 & 0.5389 \\
\hline 38 & 63 & 8 & 0.421 & 0.3245 & 0.5490 & 0.5583 \\
\hline
\end{tabular}

Table 15: Ledwina dependence measure (3.3) for each model

\begin{tabular}{|c|c|c|c|c|c|c|}
\hline patient & $\mathrm{T}_{1}$ & $\mathrm{~T}_{2}$ & LED EMP & LED BB & LED GB & LED FGM \\
\hline 1 & 8 & 16 & -1.5315 & 0.0164 & -0.0078 & 0.0088 \\
\hline 2 & 23 & 13 & -0.8038 & -3.6514 & -0.0105 & 0.0113 \\
\hline 3 & 22 & 28 & -0.7997 & 0.0326 & -0.0147 & 0.0151 \\
\hline 4 & 447 & 318 & -0.0930 & 0.0064 & -0.0355 & 0.0078 \\
\hline 5 & 30 & 12 & -0.5889 & -3.2455 & -0.0111 & 0.0117 \\
\hline 6 & 24 & 245 & 0.0484 & 0.0322 & -0.0284 & 0.0155 \\
\hline 7 & 7 & 9 & -2.0167 & 0.0118 & -0.0057 & 0.0065 \\
\hline 8 & 511 & 30 & -0.2838 & -0.0905 & -0.0228 & 0.0085 \\
\hline 9 & 53 & 196 & 0.0652 & 0.0561 & -0.0364 & 0.0208 \\
\hline 10 & 15 & 154 & 0.0788 & 0.0324 & -0.0233 & 0.0167 \\
\hline 11 & 7 & 333 & 0.1438 & 0.0126 & -0.0174 & 0.0081 \\
\hline 12 & 141 & 8 & 0.2581 & -1.2645 & -0.0139 & 0.0108 \\
\hline 13 & 96 & 38 & -0.2513 & -0.7625 & -0.0262 & 0.0209 \\
\hline 14 & 149 & 70 & -0.0256 & -0.3363 & -0.0359 & 0.0228 \\
\hline 15 & 536 & 25 & $*$ & -0.0909 & -0.0206 & 0.0075 \\
\hline 16 & 17 & 4 & $*$ & -7.8965 & -0.0053 & 0.0060 \\
\hline 17 & 185 & 117 & -0.2020 & -0.1701 & -0.0426 & 0.0219 \\
\hline 18 & 292 & 114 & -0.0455 & -0.0846 & -0.0418 & 0.0177 \\
\hline 19 & 22 & 159 & 0.0054 & 0.0390 & -0.0268 & 0.0185 \\
\hline 20 & 15 & 108 & -0.2109 & 0.0349 & -0.0214 & 0.0176 \\
\hline 21 & 152 & 562 & $*$ & 0.0262 & -0.0291 & 0.0065 \\
\hline 22 & 402 & 24 & 1.0403 & -0.1716 & -0.0226 & 0.0100 \\
\hline 23 & 13 & 66 & -0.1838 & 0.0322 & -0.0173 & 0.0162 \\
\hline 24 & 39 & 46 & -0.3166 & 0.0493 & -0.0220 & 0.0202 \\
\hline & & & & & & \\
\hline
\end{tabular}


Jorge Alberto Achcar, Jose Rafael Tovar Cuevas, Fernando A. Moala

\begin{tabular}{|c|c|c|c|c|c|c|}
\hline 25 & 12 & 40 & -0.6546 & 0.0278 & -0.0137 & 0.0141 \\
\hline 26 & 113 & 201 & 0.0804 & 0.0783 & -0.0433 & 0.0210 \\
\hline 27 & 132 & 156 & -0.1242 & 0.0870 & -0.0434 & 0.0226 \\
\hline 28 & 34 & 30 & -0.4972 & -1.8611 & -0.0176 & 0.0172 \\
\hline 29 & 2 & 25 & -2.2910 & 0.0099 & -0.0056 & 0.0062 \\
\hline 30 & 130 & 26 & -0.1982 & -0.7212 & -0.0236 & 0.0179 \\
\hline 31 & 27 & 58 & -0.4005 & 0.0450 & -0.0214 & 0.0196 \\
\hline 32 & 5 & 43 & -0.9097 & 0.0184 & -0.0102 & 0.0105 \\
\hline 33 & 152 & 30 & -0.2637 & -0.5684 & -0.0257 & 0.0183 \\
\hline 34 & 190 & 5 & $*$ & -1.1875 & -0.0115 & 0.0080 \\
\hline 35 & 119 & 8 & -0.2932 & -1.4790 & -0.0134 & 0.0111 \\
\hline 36 & 54 & 16 & -0.3475 & -1.9086 & -0.0153 & 0.0147 \\
\hline 37 & 6 & 78 & -0.6090 & 0.0221 & -0.0137 & 0.0127 \\
\hline 38 & 63 & 8 & -0.6750 & -2.4530 & -0.0115 & 0.0111 \\
\hline
\end{tabular}

Figure 5 shows the plots of the Ledwina dependence measures considering each fitted model. From these plots, it is possible to see that the BB bivariate exponential distribution is better in comparison with the other copula models, since the plot of the estimated Ledwina BB measure is more close to the empirical Ledwina measure using the LY non-parametric estimator, although the data set has a very small sample size. The two copula models (GB and FGM models) give similar fit for the data set.

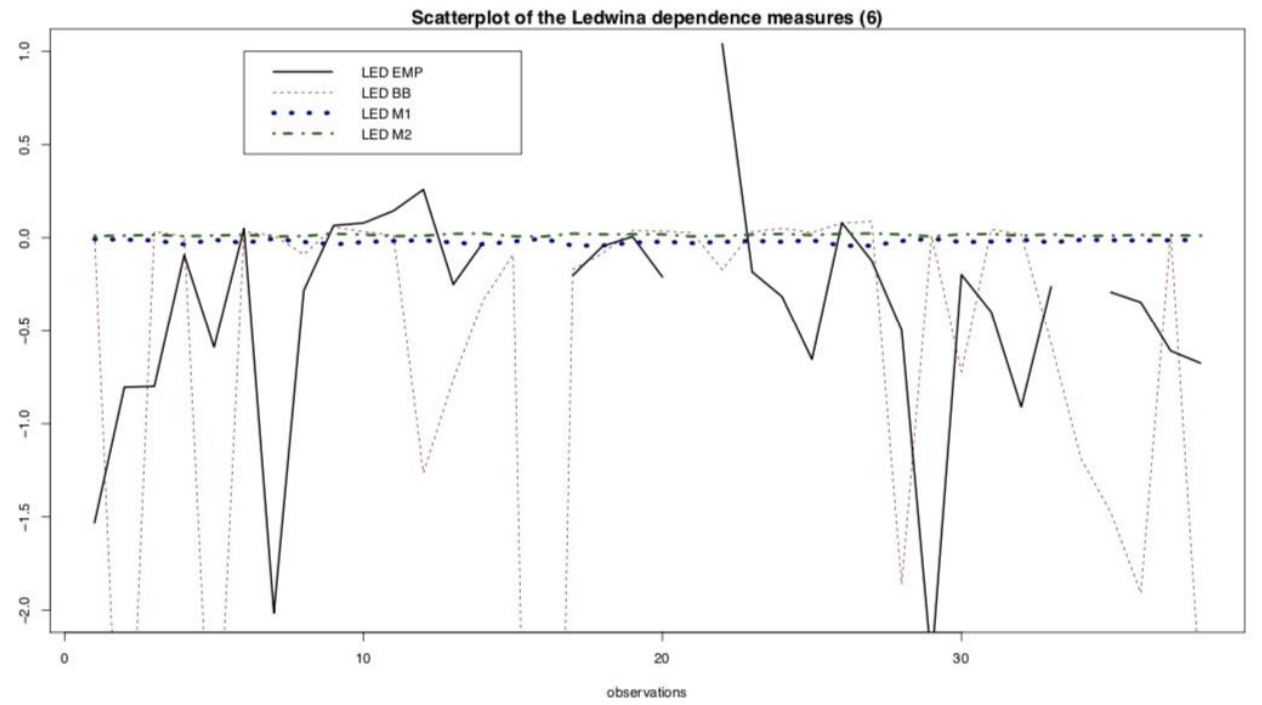

Figure 5: Plots of the Ledwina dependence measures for each fitted model

\subsection{Application 5: Thyroid cancer data}

As our last application, we used a medical data set related to differentiated cancer o thyroid. In this data set, there are the observations of 15 patients treated by the head and neck surgeon Gabriel Sanchez de Guzman who works in a level IV hospital located in Bogotá Colombia. Each patient received surgery to remove the thyroid gland and then was treated with redioiodine 

lifetime data in presence of censored data

I-131. Each individual was followed after finalized the therapy until to be observed a thyroglobulin level greater than or equal $2.0 \mathrm{ng} / \mathrm{ml}$ which is an indicator of the presence of recurrence/persistence of the cancer. In this way, the time recorded for each patient is the recurrence/persistence time (in months). Fourteen individuals presented two recurrence/persistence events and at the date of the last control, one individual did not present recurrence/persistence (censored time). The data were analysed in the same form as considered for the other data sets. For the Bayesian analysis, we considered the same prior distributions for the parameters that were assumed to analyse the infection for Kidney data and, to get the posterior summaries of interest the same simulation approach was used. Table 16 presents the posterior summaries of interest and the Kaplan-Meier (KM) estimates for the marginal survival functions of the lifetimes are reported in Table 17.

Table 16: Posterior means and posterior standard deviation (in parenthesis) for the parameters of the three models

\begin{tabular}{|c|c|c|c|c|c|}
\hline model & $\lambda_{1}$ & & $\lambda_{2}$ & & $\lambda_{3}$ \\
\hline BB & $0.000004\left(1.0 \mathrm{e}^{-12}\right)$ & & $0.00009\left(1.0 \mathrm{e}^{-12}\right)$ & & $0.0026\left(1.0 \mathrm{e}^{-12}\right)$ \\
\hline Copula & $\lambda_{1}$ & $\mathrm{p}_{1}$ & $\lambda_{2}$ & $p_{2}$ & $\theta$ \\
\hline GB & $850.7(29.75)$ & $0.7478(0.1448)$ & $849(29.02)$ & $1.47(0.2765)$ & $0.2002(0.188)$ \\
\hline FGM & $846.7(28.63)$ & $0.7677(0.1521)$ & $855.8(28.21)$ & $1.504(0.2876)$ & $0.4288(0.2824)$ \\
\hline
\end{tabular}

Table 17: Estimators for the joint survival function $S\left(t_{1} t_{2}\right)$

\begin{tabular}{|c|c|c|c|c|c|c|c|c|}
\hline pair & $t_{1}$ & $t_{2}$ & $\mathrm{LY}$ & $\mathrm{KM} \mathrm{S}\left(t_{1}\right)$ & $\mathrm{KM} \mathrm{S}\left(t_{2}\right)$ & $\mathrm{BB}$ & GB & FGM \\
\hline 1 & 88 & 460 & 0.5333 & 0.7333 & 0.7143 & 0.3045 & 0.5391 & 0.5721 \\
\hline 2 & 3634 & 645 & 0.0000 & 0.0000 & $*$ & 0.1174 & 0.0214 & 0.0334 \\
\hline 3 & 1634 & 2304 & 0.0000 & 0.1333 & 0.0000 & 0.0023 & 0.0017 & 0.0049 \\
\hline 4 & 875 & 1353 & 0.0667 & 0.2667 & 0.0714 & 0.0282 & 0.0356 & 0.0617 \\
\hline 5 & 322 & 50 & 0.4000 & 0.4000 & 0.9286 & 0.8475 & 0.6006 & 0.6096 \\
\hline 6 & 925 & 455 & 0.1333 & 0.2000 & 0.7857 & 0.2800 & 0.2118 & 0.2528 \\
\hline 7 & 2900 & 420 & 0.0667 & 0.0667 & 0.8571 & 0.2331 & 0.0511 & 0.0642 \\
\hline 8 & 713 & 560 & 0.0667 & 0.3333 & 0.5714 & 0.2195 & 0.2203 & 0.2698 \\
\hline 9 & 122 & 699 & 0.2667 & 0.6000 & 0.4286 & 0.1642 & 0.3572 & 0.3959 \\
\hline 10 & 243 & 519 & 0.2667 & 0.4667 & 0.6429 & 0.2572 & 0.3976 & 0.4440 \\
\hline 11 & 92 & 672 & 0.2667 & 0.6667 & 0.5000 & 0.1766 & 0.3923 & 0.4274 \\
\hline 12 & 48 & 1238 & 0.1333 & 0.9333 & 0.2143 & 0.0414 & 0.1491 & 0.1628 \\
\hline 13 & 136 & 974 & 0.1333 & 0.5333 & 0.3571 & 0.0809 & 0.2130 & 0.2459 \\
\hline 14 & 81 & 1270 & 0.0667 & 0.8000 & 0.2143 & 0.0380 & 0.1296 & 0.1467 \\
\hline 15 & 66 & 1138 & $*$ & 0.8667 & 0.2857 & 0.0534 & 0.1756 & 0.1947 \\
\hline
\end{tabular}

From the results showed in Tables 17 and 18 and the plots presented in Figure 6, the model with the best fit is the GB model since for this model we observed values of the Ledwima measure more close to the empirical Ledwina measure using the LY non-parametric estimator. It is important to point out that in this example there is a very small sample size, usually a great difficult to fit a parametrical model, but as illustrative purposes, it was possible to fit marginal Weibull distributions and a dependence structure type Gumbel Barnett. 
Table 18: Ledwina dependence measure (3.3)for each model

\begin{tabular}{|c|c|c|c|c|c|c|}
\hline Pair & $t_{1}$ & $t_{2}$ & LED EMP & LED BB & LED GB & LED FGM \\
\hline 1 & 88 & 460 & -0.0227272 & -1.1928977 & 0.0067614 & 0.1755114 \\
\hline 2 & 3634 & 645 & $*$ & $*$ & $*$ & $*$ \\
\hline 3 & 1634 & 2304 & $*$ & $*$ & $*$ & $*$ \\
\hline 4 & 875 & 1353 & 0.4432026 & 0.0947547 & 0.1612955 & 0.3977240 \\
\hline 5 & 322 & 50 & 0.2182179 & 3.8801868 & 1.8597619 & 1.9334105 \\
\hline 6 & 925 & 455 & -0.1666667 & 0.7500000 & 0.3237500 & 0.5800000 \\
\hline 7 & 2900 & 420 & 0.1048284 & 2.0676107 & -0.0782807 & 0.0758565 \\
\hline 8 & 713 & 560 & -0.5773503 & 0.0844375 & 0.0879016 & 0.3022429 \\
\hline 9 & 122 & 699 & 0.1111111 & -0.3158333 & 0.4883333 & 0.6495833 \\
\hline 10 & 243 & 519 & -0.1889823 & -0.2292355 & 0.3677594 & 0.5650569 \\
\hline 11 & 92 & 672 & -0.1889823 & -0.5719548 & 0.3452233 & 0.4944720 \\
\hline 12 & 48 & 1238 & -0.5345225 & -1.4561060 & -0.3765043 & -0.2391988 \\
\hline 13 & 136 & 974 & -0.1889822 & -0.4119765 & 0.1497684 & 0.2896625 \\
\hline 14 & 81 & 1270 & -0.2941742 & -0.5051461 & 0.1686599 & 0.2944194 \\
\hline 15 & 66 & 1138 & $*$ & -1.1819818 & -0.3692746 & -0.2422164 \\
\hline
\end{tabular}

Scatterplot of the Ledwina dependence measures (6)

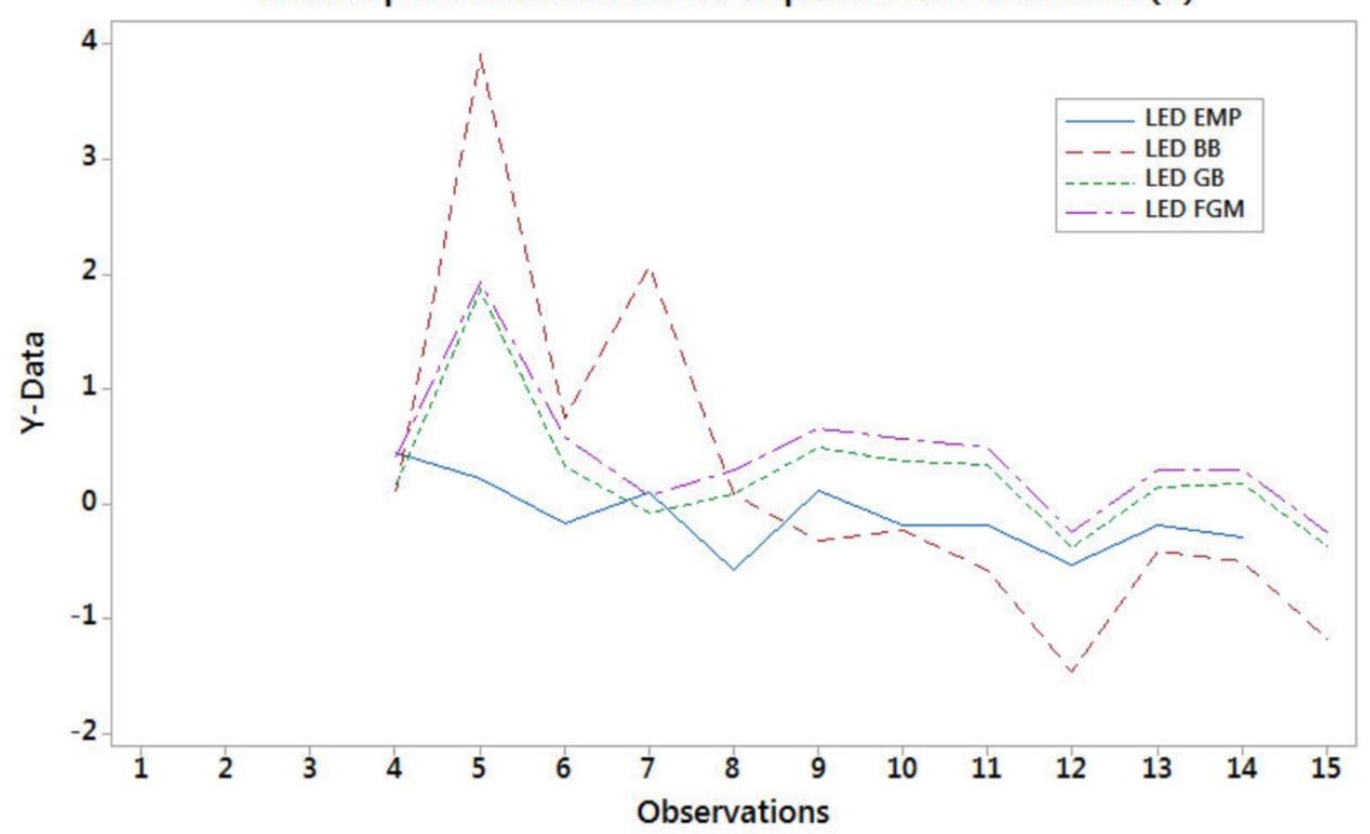

Figure 6: Plots of the Ledwina dependence measures for each fitted model

\section{Concluding remarks}

Usually in applications with bivariate lifetime data in presence of censoring, the choice of an appropriate parametrical distribution is not easy, since each assumed parametrical model has 
a different dependence structure. The proposed graphical method based on the Ledwina dependence measure introduced in this paper could be very useful to decide by an appropriate bivariate lifetime distribution in applications with bivariate data sets in presence of univariate censoring. In this way, the LY non-parametric estimator for the survival function that is a generalization of the popular Kaplan-Meier product limit estimator could be a starting point in each application. Plots of the Ledwina dependence measure considering the LY estimator could be compared to the plots of the Ledwina dependence measure assuming any parametrical lifetime distribution, implying in an useful graphical tool to be used in applications considering bivariate lifetime data. It is important to point out that we could use our proposed methodology in applications of bivariate lifetime analysis in presence of univariate censoring mechanism considering any parametrical model introduced in the literature to be fitted by bivariate lifetime data. Each proposed parametrical model have some physical interpretations as the degree of dependence associated to each distribution, but in applications usually it is difficult for a statistician to decide if the proposed model is good in terms of good fit. From the obtained results of this study it was observed that in practical applications standard copula based models should be used with caution, since every copula function implies in different dependence structures which could not capture the existing dependence structure for the dataset if the model is not appropriate. Finally, it is important to point out that our proposed graphical approach is useful in discrimination of proposed models but also in the indication of the good fit of a proposed model for bivariate lifetime data in presence of censored data. As alternative to our methodology, the popular existing model selection criteria AIC (Akaike information criterion), BIC (Bayesian information criterion) and DIC (deviance information criterion) only indicates the best among different models, but all proposed models could be wrong.

\section{Acknowledgments}

The authors are grateful to Gabriel Sánchez de Guzmán, a head and neck surgeon who works in the Hospital San Ignacio in Bogotá, and who kindly provided the data set used in our fifth application. We also are grateful to Jennyfer Portilla Yela by her valuable help to obtain the results of the last application. We would like to thank the Editor-in-Chief and the referees for their careful reading and for the comments which greatly improved the paper.

\section{References}


[1] Achcar, J.A.; Leandro, R.A. (1998). Use of Markov Chain Monte Carlo methods in a Bayesian analysis of the Block and Basu bivariate exponential distribution. Annals of the Institute of Statistical Mathematics, 50, 3, 403-416.

[2] Achcar, J.A.; Santos, C.A. (2011). A Bayesian Analysis for the Block-Basu Bivariate Exponential Distribution in the Presence of Covariates and Censored Data. Journal of Applied Statistics, v. 38, p. 2213-2223.

[3] Arnold, B.C.; Strauss, D. (1988). Bivariate distributions with exponential conditionals. Journal of the American Statistical Association, 83, 402, 522-527.

[4] Bairamov, I.; Kotz, S.; Kozubowski, T.J. (2003). A new measure of linear local dependence. Statistics, 37, 243-258.

[5] Balakrishnan, N.; Lai, C.D. (2009). Continuous Bivariate Distributions, Springer, Dordrecht.

[6] Barnett, V. (1980). Some bivariate uniform distributions. Communications in Statistics - Theory and Methods, 9, 453-461.

[7] Bjerve, S.; Doksum, K. (1993). Correlation curves: measures of association as function of covariate values. The Annals of Statistics, 21, 890-902.

[8] Block, H.W.; Basu, A.P. (1974). A continuous bivariate exponential extension. Journal of American Statistical Association, 69, 1031-1037.

[9] Campbell, G. (1981). Nonparametric bivariate estimation with randomly censored data. Biometrika, 68, 417-422.

[10] Campbell, G.; Foldes, A. (1982). Large sample properties of nonparametric bivariate estimators with censored data. In Nonparametric Statistical Inference, Colloquia Mathematica-Societatis Janos Bolyai, Ed. B.V. Gnedenko, M.L. Puri and I. Vincze, pp 103-122, Amsterdam: NorthHolland.

[11] Chib,S.; Greenberg, E. (1995). Understanding the Metropolis-Hastings algorithm. American Statistician, 49 , pp. 327-335.

[12] Dabrowska, D.M. (1988). Kaplan-Meier estimate on the plane. Annals of Statistics, 16, 14751489.

[13] Davarzani , N.; Achcar, J.A.; Smirnov, E.N.; Peeters, R. (2015). Bivariate lifetime geometric distribution in presence of cure fractions, Journal of Data Science 13, 755-770.

[14] Downton, F. (1970). Bivariate exponential distributions in reliability theory. Journal of the Royal Statistical Society, B, 32, 408-417.

[15] Drouet M.D.; Kotz, S. (2001). Correlation and Dependence. Imperial College Press, London.

[16] Durante, F.; Sempi, C. (2015). Principles of copula theory. CRC Press, 332 pages.

[17] Flores, Y.S. (2016) General multivariate dependence using associated copulas, Revstat Statistical Journal, 14 , No. 1, pp. 1-28.

[18] Freund, J.E. (1961). A bivariate extension of the exponential distribution. Journal of the American Statistical Association, 56, 971-977. 
[19] Gelfand, A.E.; Smith, A.F.M. (1990). Sampling-based approaches to calculating marginal densities. J.Am. Statist. Assoc. 85, pp. 398-410.

[20] Goethals,K.; Paul Janssen, P.; Duchateau, L. (2008). Frailty models and copulas: similarities and differences. Journal of Applied Statistics, Vol. 35, No. 9, 1071-1079.

[21] Gumbel, E.J. (1960). Bivariate exponential distributions. Journal of the American Statistical Association, 55, 698-707.

[22] Hanley, J.A.; Parnes, M.N. (1983). Nonparametric estimation of a multivariate distribution in the presence of censoring. Biometrics, 39, 129-139.

[23] Hawkes, A.G. (1972). A bivariate exponential distribution with applications to reliability. Journal of the Royal Statistical Society, B, 34, 129-131.

[24] Holt, J.D.; Prentice, R.L. (1974). Survival analysis in twin studies and matched pairs experiments. Biometrika, 61, 17-30.

[25] Hougaard, P. (1986). A class of multivariate failure time distributions. Biometrika, 3, 73, 671678.

[26] Icuma, T.R.; Buzatto, I.P.C.; Tiezzi, D.G.; Achcar, J.A.; Davarzani, N. (2016). Use of Bivariate Lifetime Distributions Assuming Continuous or Discrete Data Applied to Patients with Breast Cancer, Journal of Data Science, 14, 657-680.

[27] Jogdeo, K. (1982). Dependence, concepts of, in: S. Kotz, N.L. Johnson (Eds), Encyclopedia of Statistical Sciences, Vol. 2. Wiley, New York, pp. 324- 334.

[28] Kaplan, E.L.; Meier, P. (1958). Nonparametric estimation from incomplete observations. Journal of the American Statistical Association, 53 (282): 457- 481。

[29] Karvanen, J.; Saarela, O; Kuulasmaa, K. (2010). Nonparametric Multiple Imputation of Left Censored Event Times in Analysis of Follow-up Data. Journal of Data Science, 8, 151-172.

[30] Klein J.P.; Moeschberger, M.L. (1997). Survival Analysis: Techniques for Censored and Truncated Data. Springer, Berlin.

[31] Kowalczyk, T.; Pleszczynska, E. (1997). Monotonic dependence functions of bivariate distributions. The Annals of Statistics, 5, 1221-1227.

[32] Lancaster, H.O. (1982). Dependence, measures and indices of, in: S. Kotz, N.L. Johnson (Eds), Encyclopedia of Statistical Sciences, Vol. 2. Wiley, New York, pp. 334-339.

[33] Langberg, N.A.; Shaked, M. (1982). On the identifiability of multivariate life distributions. Annals of Probability, 10,773-779.

[34] Lawless, J.F. (1982). Statistical Models and Methods for Lifetime Data. Wiley, New York, 1982.

[35] Ledwina, T. (2015). Visualizing association structure in bivariate copulas using new dependence function. In: Stochastic models, Statistics and their applications, Ed: Steland, A.; Rafajlowicz, E.; Szajowski, K;. Springer Proceedings in Mathematics and Statistics, 19-27. 
[36] Lin, D.Y.; Ying, Z. (1993). A simple nonparametric estimator of the bivariate survival function under univariate censoring. Biometrika, 80, 3, 573-581.

[37] Marshall, A.W.; Olkin, I. (1967). A generalized bivariate exponential distribution. Journal of Applied Probability, 4, 291-302.

[38] Martinez, E.Z.; Achcar, J.A.; Peres, M.V.O.; Queiroz, J.A.M. (2016). A brief note on the simulation of survival data with a desired percentage of right-censored data. Journal of Data Science, 14, 701-712.

[39] McGilchrist, C.A. and Aisbett, C.W. (1991). Regression with frailty in survival analysis, Biometrics, 47, 461 466. pp. 461466.

[40] Morgenstern, D. (1956). Einfachebeispielezweidimensionalerverteilungen. Mit- teilingsblatt fur MathematisheStatistik, 8, 234-235.

[41] Nelsen, R.B. (2006). An Introduction to Copulas. Springer, New York.

[42] Prentice, R.L.; Cai, J. (1992). Covariance and survival function estimation using censored multivariate failure time data. Biometrika, 79, 495-512.

[43] Pruitt, R.C. (1993). Small sample comparisons of five bivariate survival estimators. Journal of Statistical Computing and Simulation, 45,147-167.

[44] Sarkar, S.K. (1987). A continuous bivariate exponential distribution. Journal of the American Statistical Association, 82, 667-675.

[45] Sklar, A. (1959). Fonctions de r partition n dimensions et leursmarges. Publ. Inst. Stat. Univ. Paris, $8,229-231$.

[46] Spiegelhalter, D.J., Thomas, A., Best, N.G., Lunn, D. (2003). WinBugs user manual, version 1.4. MRC Biostatistics Unit Cambridge, U.K.

[47] Tsai, W.Y.; Leurgans, S.; Crowley, J. (1986). Nonparametric estimation of a bivariate survival function in the presence of censoring. Annals of Statistics, 13, 1351-1365.

[48] Wang., J. (2011). Estimation of Lifetime Distribution with Missing Censoring. Journal of Data Science, 9, 331-343.

Table 6: Estimators for the joint survival function $\mathrm{S}\left(t_{1} t_{2}\right)$

\begin{tabular}{|c|c|c|c|c|c|c|}
\hline pairs & $\mathrm{t}_{1}$ & $\mathrm{t}_{2}$ & LY & BB & GB & FGM \\
\hline 1 & 1 & 1 & 1.00000 & 0.998761 & 0.976321 & 0.977043 \\
\hline 2 & 2 & 2 & 1.00000 & 0.997523 & 0.967970 & 0.968942 \\
\hline 3 & 10 & 10 & 0.98507 & 0.987677 & 0.932058 & 0.934001 \\
\hline 4 & 16 & 16 & 0.97744 & 0.980356 & 0.914288 & 0.916662 \\
\hline 5 & 35 & 35 & 0.87121 & 0.957528 & 0.872530 & 0.875826 \\
\hline 6 & 48 & 48 & 0.83969 & 0.942217 & 0.850027 & 0.853776 \\
\hline 7 & 53 & 53 & 0.83077 & 0.936393 & 0.842154 & 0.846055 \\
\hline 8 & 62 & 62 & 0.82946 & 0.926001 & 0.828827 & 0.832979 \\
\hline 9 & 38 & 63 & 0.85156 & 0.931273 & 0.835869 & 0.839677 \\
\hline 10 & 73 & 73 & 0.81102 & 0.913456 & 0.813748 & 0.818174 \\
\hline
\end{tabular}



lifetime data in presence of censored data

\begin{tabular}{|l|l|l|l|l|l|l|}
\hline 11 & 29 & 74 & 0.88889 & 0.923753 & 0.827687 & 0.831398 \\
\hline 12 & 16 & 79 & 0.97600 & 0.922625 & 0.829971 & 0.833308 \\
\hline 13 & 10 & 80 & 0.98374 & 0.923268 & 0.833989 & 0.837051 \\
\hline 14 & 21 & 80 & 0.95122 & 0.920463 & 0.825625 & 0.829142 \\
\hline 15 & 38 & 84 & 0.86179 & 0.912622 & 0.813298 & 0.817267 \\
\hline 16 & 10 & 84 & 0.96748 & 0.919717 & 0.829805 & 0.832867 \\
\hline 17 & 86 & 86 & 0.81978 & 0.898849 & 0.797298 & 0.802011 \\
\hline 18 & 93 & 93 & 0.79361 & 0.891081 & 0.788953 & 0.793807 \\
\hline 19 & 70 & 95 & 0.83787 & 0.895043 & 0.792377 & 0.796955 \\
\hline 20 & 97 & 97 & 0.77538 & 0.886672 & 0.784325 & 0.789256 \\
\hline 21 & 18 & 100 & 0.89210 & 0.903646 & 0.807512 & 0.810942 \\
\hline 22 & 21 & 100 & 0.87542 & 0.902897 & 0.805654 & 0.809197 \\
\hline 23 & 21 & 105 & 0.88175 & 0.898558 & 0.800929 & 0.804470 \\
\hline 24 & 105 & 105 & 0.76190 & 0.877920 & 0.775352 & 0.780429 \\
\hline 25 & 105 & 105 & 0.76190 & 0.877920 & 0.775352 & 0.780429 \\
\hline 26 & 107 & 107 & 0.76013 & 0.875745 & 0.773163 & 0.778276 \\
\hline 27 & 110 & 110 & 0.75832 & 0.872494 & 0.769920 & 0.775084 \\
\hline 28 & 30 & 120 & 0.77575 & 0.883464 & 0.782743 & 0.786568 \\
\hline 29 & 67 & 121 & 0.72136 & 0.873627 & 0.769570 & 0.774207 \\
\hline 30 & 72 & 121 & 0.72136 & 0.872420 & 0.768315 & 0.773032 \\
\hline 31 & 28 & 121 & 0.75832 & 0.883101 & 0.782787 & 0.786548 \\
\hline 32 & 88 & 122 & 0.69497 & 0.867731 & 0.763810 & 0.768764 \\
\hline 33 & 122 & 122 & 0.70833 & 0.859607 & 0.757383 & 0.762743 \\
\hline 34 & 128 & 128 & 0.68805 & 0.853235 & 0.751356 & 0.756808 \\
\hline 35 & 129 & 129 & 0.68565 & 0.852178 & 0.750366 & 0.755833 \\
\hline 36 & 25 & 140 & 0.68565 & 0.867801 & 0.768123 & 0.771731 \\
\hline 37 & 153 & 153 & 0.60017 & 0.827191 & 0.727735 & 0.733530 \\
\hline 38 & 36 & 155 & 0.60941 & 0.852752 & 0.751312 & 0.755234 \\
\hline 39 & 28 & 156 & 0.63428 & 0.853817 & 0.753868 & 0.757523 \\
\hline 40 & 162 & 162 & 0.59036 & 0.818011 & 0.719749 & 0.725655 \\
\hline 41 & 162 & 162 & 0.59036 & 0.818011 & 0.719749 & 0.725655 \\
\hline & & & & & & \\
\hline
\end{tabular}

\begin{tabular}{|c|c|c|c|c|c|c|}
\hline 42 & 164 & 164 & 0.58696 & 0.815984 & 0.718007 & 0.723937 \\
\hline 43 & 22 & 172 & 0.64182 & 0.842151 & 0.744574 & 0.747906 \\
\hline 44 & 183 & 130 & 0.66838 & 0.838705 & 0.741867 & 0.747828 \\
\hline 45 & 194 & 94 & 0.80482 & 0.865595 & 0.771873 & 0.777579 \\
\hline 46 & 195 & 195 & 0.54244 & 0.785213 & 0.692403 & 0.698667 \\
\hline 47 & 168 & 200 & 0.52815 & 0.787299 & 0.692272 & 0.698269 \\
\hline 48 & 222 & 123 & 0.69842 & 0.835254 & 0.743022 & 0.749215 \\
\hline 49 & 226 & 226 & 0.47783 & 0.755602 & 0.669036 & 0.675577 \\
\hline 50 & 243 & 243 & 0.44130 & 0.739841 & 0.657018 & 0.663693 \\
\hline 51 & 248 & 100 & 0.78656 & 0.847737 & 0.760154 & 0.766256 \\
\hline 52 & 262 & 262 & 0.40866 & 0.722614 & 0.644173 & 0.650982 \\
\hline 53 & 265 & 210 & 0.51147 & 0.759077 & 0.675467 & 0.682325 \\
\hline
\end{tabular}


Jorge Alberto Achcar, Jose Rafael Tovar Cuevas, Fernando A. Moala

\begin{tabular}{|c|c|c|c|c|c|c|}
\hline 54 & 269 & 120 & 0.73790 & 0.826740 & 0.740650 & 0.747102 \\
\hline 55 & 276 & 81 & 0.83608 & 0.856590 & 0.775218 & 0.781151 \\
\hline 56 & 318 & 140 & 0.66584 & 0.799965 & 0.720123 & 0.727029 \\
\hline 57 & 20 & 348 & 0.47398 & 0.711158 & 0.637026 & 0.638858 \\
\hline 58 & 350 & 350 & 0.41142 & 0.647912 & 0.591254 & 0.598527 \\
\hline 59 & 32 & 360 & 0.43427 & 0.700654 & 0.625950 & 0.628329 \\
\hline 60 & 363 & 363 & 0.40518 & 0.637552 & 0.584202 & 0.591525 \\
\hline 61 & 371 & 184 & 0.56299 & 0.755579 & 0.684093 & 0.691559 \\
\hline 62 & 52 & 380 & 0.41051 & 0.683492 & 0.610223 & 0.613218 \\
\hline 63 & 390 & 390 & 0.40417 & 0.616560 & 0.570082 & 0.577499 \\
\hline 64 & 392 & 122 & 0.72302 & 0.797130 & 0.728613 & 0.735682 \\
\hline 65 & 393 & 100 & 0.79415 & 0.813875 & 0.747320 & 0.754072 \\
\hline 66 & 414 & 414 & 0.40886 & 0.598481 & 0.558085 & 0.565572 \\
\hline 67 & 417 & 417 & 0.40213 & 0.596259 & 0.556620 & 0.564115 \\
\hline 68 & 418 & 220 & 0.49720 & 0.720364 & 0.657562 & 0.665402 \\
\hline 69 & 431 & 431 & 0.40103 & 0.585997 & 0.549879 & 0.557409 \\
\hline 70 & 466 & 119 & 0.77464 & 0.782874 & 0.725914 & 0.733231 \\
\hline 71 & 469 & 90 & 0.82636 & 0.804209 & 0.750977 & 0.757794 \\
\hline 72 & 39 & 487 & 0.41953 & 0.618722 & 0.565697 & 0.567351 \\
\hline 73 & 487 & 76 & 0.83907 & 0.810887 & 0.763165 & 0.769702 \\
\hline 74 & 491 & 180 & 0.59117 & 0.733275 & 0.678928 & 0.686947 \\
\hline 75 & 515 & 515 & 0.41910 & 0.528031 & 0.512445 & 0.520122 \\
\hline 76 & 526 & 121 & 0.76674 & 0.768188 & 0.720510 & 0.728061 \\
\hline 77 & 547 & 130 & 0.75065 & 0.757076 & 0.712079 & 0.719827 \\
\hline 78 & 583 & 583 & 0.42573 & 0.485333 & 0.485372 & 0.493101 \\
\hline 79 & 641 & 641 & 0.41813 & 0.451653 & 0.464179 & 0.471915 \\
\hline 80 & 653 & 653 & 0.41028 & 0.444983 & 0.459989 & 0.467723 \\
\hline 81 & 677 & 150 & 0.66507 & 0.715724 & 0.690040 & 0.698451 \\
\hline 82 & 716 & 716 & 0.40945 & 0.411544 & 0.438990 & 0.446695 \\
\hline 83 & 732 & 732 & 0.40099 & 0.403459 & 0.433907 & 0.441600 \\
\hline 84 & 781 & 781 & 0.39221 & 0.379675 & 0.418916 & 0.426564 \\
\hline 85 & 845 & 845 & 0.38310 & 0.350708 & 0.400543 & 0.408114 \\
\hline 86 & 847 & 847 & 0.37363 & 0.349840 & 0.399990 & 0.407558 \\
\hline 87 & 848 & 155 & 0.64094 & 0.678208 & 0.678789 & 0.687729 \\
\hline 88 & 860 & 860 & 0.37120 & 0.344246 & 0.396420 & 0.403970 \\
\hline 89 & 29 & 932 & 0.37893 & 0.403927 & 0.423999 & 0.421952 \\
\hline 90 & 957 & 957 & 0.36857 & 0.305233 & 0.371268 & 0.378663 \\
\hline 91 & 1030 & 210 & 0.54604 & 0.610690 & 0.635842 & 0.645797 \\
\hline 92 & 1063 & 240 & 0.46194 & 0.587880 & 0.617116 & 0.627355 \\
\hline 93 & 1074 & 120 & 0.76771 & 0.656767 & 0.696319 & 0.705151 \\
\hline 94 & 1111 & 1111 & 0.38271 & 0.252173 & 0.336008 & 0.343104 \\
\hline 95 & 1136 & 140 & 0.70116 & 0.632901 & 0.678927 & 0.688257 \\
\hline 96 & 1156 & 180 & 0.61263 & 0.605790 & 0.650586 & 0.660535 \\
\hline 97 & 1182 & 112 & 0.80118 & 0.641288 & 0.698957 & 0.707789 \\
\hline 98 & 1199 & 91 & 0.84393 & 0.650949 & 0.716128 & 0.724417 \\
\hline 99 & 1238 & 250 & 0.47865 & 0.553489 & 0.606185 & 0.616906 \\
\hline 100 & 1258 & 120 & 0.81932 & 0.622480 & 0.690088 & 0.699224 \\
\hline
\end{tabular}



lifetime data in presence of censored data

\begin{tabular}{|c|c|c|c|c|c|c|}
\hline 101 & 1279 & 1279 & 0.43307 & 0.204751 & 0.302861 & 0.309584 \\
\hline 102 & 1298 & 1298 & 0.42069 & 0.199984 & 0.299410 & 0.306089 \\
\hline 103 & 25 & 1324 & 0.43307 & 0.277004 & 0.339842 & 0.335593 \\
\hline 104 & 1330 & 96 & 0.86614 & 0.623527 & 0.707473 & 0.716087 \\
\hline 105 & 1356 & 1356 & 0.43307 & 0.186106 & 0.289212 & 0.295755 \\
\hline 106 & 1363 & 200 & 0.64262 & 0.559590 & 0.631719 & 0.642302 \\
\hline 107 & 1377 & 123 & 0.86614 & 0.599475 & 0.684079 & 0.693462 \\
\hline 108 & 1384 & 200 & 0.68694 & 0.556165 & 0.631135 & 0.641758 \\
\hline 109 & 1433 & 236 & 0.58774 & 0.529865 & 0.608648 & 0.619690 \\
\hline 110 & 1447 & 220 & 0.64158 & 0.535758 & 0.617459 & 0.628397 \\
\hline 111 & 1470 & 180 & 0.69957 & 0.552705 & 0.641371 & 0.651906 \\
\hline 112 & 1496 & 307 & 0.55433 & 0.486347 & 0.569905 & 0.581443 \\
\hline 113 & 1499 & 1499 & 0.54134 & 0.155866 & 0.266052 & 0.272254 \\
\hline 114 & 1527 & 1527 & 0.52721 & 0.150547 & 0.261819 & 0.267954 \\
\hline 115 & 1535 & 1535 & 0.51181 & 0.149061 & 0.260627 & 0.266743 \\
\hline 116 & 1562 & 1562 & 0.49494 & 0.144153 & 0.256657 & 0.262707 \\
\hline 117 & 1568 & 1568 & 0.47637 & 0.143084 & 0.255785 & 0.261822 \\
\hline 118 & 1602 & 139 & 0.86614 & 0.552720 & 0.665831 & 0.675876 \\
\hline 119 & 1631 & 150 & 0.76990 & 0.542352 & 0.657257 & 0.667559 \\
\hline 120 & 1674 & 1674 & 0.50949 & 0.125461 & 0.241036 & 0.246818 \\
\hline 121 & 1799 & 140 & 0.86614 & 0.521050 & 0.660156 & 0.670475 \\
\hline 122 & 1829 & 1829 & 0.51968 & 0.103523 & 0.221453 & 0.226870 \\
\hline 123 & 1843 & 1843 & 0.49494 & 0.101741 & 0.219790 & 0.225174 \\
\hline 124 & 1850 & 1850 & 0.46638 & 0.100862 & 0.218965 & 0.224333 \\
\hline 125 & 1857 & 260 & 0.50524 & 0.457300 & 0.585622 & 0.597599 \\
\hline 126 & 1870 & 230 & 0.62992 & 0.468649 & 0.601678 & 0.613426 \\
\hline 127 & 2024 & 180 & 0.77952 & 0.469450 & 0.628166 & 0.639505 \\
\hline 128 & 2133 & 250 & 0.67366 & 0.425480 & 0.585365 & 0.597683 \\
\hline 129 & 2140 & 220 & 0.75787 & 0.436793 & 0.601781 & 0.613810 \\
\hline 130 & 2204 & 2204 & 0.74240 & 0.065027 & 0.182018 & 0.186597 \\
\hline 131 & 2218 & 2218 & 0.72179 & 0.063907 & 0.180729 & 0.185279 \\
\hline 132 & 2252 & 150 & 0.86614 & 0.451370 & 0.643174 & 0.654218 \\
\hline 133 & 2409 & 2409 & 0.86613 & 0.050430 & 0.164254 & 0.168414 \\
\hline 134 & 2430 & 2430 & 0.86614 & 0.049134 & 0.162561 & 0.166679 \\
\hline 135 & 2506 & 2506 & 0.86613 & 0.044715 & 0.156615 & 0.160587 \\
\hline 136 & 2569 & 2569 & 0.86618 & 0.041355 & 0.151892 & 0.155745 \\
\hline 137 & 2640 & 2640 & $*$ & 0.037870 & 0.146782 & 0.150504 \\
\hline & & & & & & \\
\hline
\end{tabular}

Table 7: Ledwina dependence measure (3.3)for each model

\begin{tabular}{|c|c|c|c|c|c|c|}
\hline Pair & $t_{1}$ & $t_{2}$ & LED EMP & LED BB & LED GB & LED FGM \\
\hline 1 & 1 & 1 & $*$ & 0.193353 & -0.001011 & 0.003020 \\
\hline 2 & 2 & 2 & $*$ & 0.193299 & -0.001439 & 0.004299 \\
\hline 3 & 10 & 10 & $*$ & 0.192865 & -0.003247 & 0.009632 \\
\hline 4 & 16 & 16 & $*$ & 0.192540 & -0.004110 & 0.012117 \\
\hline 5 & 35 & 35 & $*$ & 0.191512 & -0.006062 & 0.017565 \\
\hline 6 & 48 & 48 & $*$ & 0.190811 & -0.007077 & 0.020294 \\
\hline
\end{tabular}


Jorge Alberto Achcar, Jose Rafael Tovar Cuevas, Fernando A. Moala

\begin{tabular}{|c|c|c|c|c|c|c|}
\hline 7 & 53 & 53 & $*$ & 0.190541 & -0.007427 & 0.021216 \\
\hline 8 & 62 & 62 & $*$ & 0.190056 & -0.008015 & 0.022743 \\
\hline 9 & 38 & 63 & $*$ & 0.158984 & -0.007458 & 0.021245 \\
\hline 10 & 73 & 73 & $*$ & 0.189464 & -0.008672 & 0.024417 \\
\hline 11 & 29 & 74 & $*$ & 0.140135 & -0.007529 & 0.021363 \\
\hline 12 & 16 & 79 & 0.46131 & 0.124191 & -0.006996 & 0.019877 \\
\hline 13 & 10 & 80 & 0.59515 & 0.121273 & -0.006516 & 0.018555 \\
\hline 14 & 21 & 80 & 0.88159 & 0.128670 & -0.007335 & 0.020793 \\
\hline 15 & 38 & 84 & 0.62626 & 0.145115 & -0.008186 & 0.023053 \\
\hline 16 & 10 & 84 & 0.66211 & 0.121242 & -0.006619 & 0.018808 \\
\hline 17 & 86 & 86 & 0.50509 & 0.188766 & -0.009381 & 0.026182 \\
\hline 18 & 93 & 93 & 0.34641 & 0.188391 & -0.009738 & 0.027054 \\
\hline 19 & 70 & 95 & 0.76194 & 0.168492 & -0.009377 & 0.026104 \\
\hline 20 & 97 & 97 & -1.21710 & 0.188176 & -0.009935 & 0.027530 \\
\hline 21 & 18 & 100 & 0.48801 & 0.122142 & -0.007687 & 0.021586 \\
\hline 22 & 21 & 100 & 0.48869 & 0.123999 & -0.007878 & 0.022099 \\
\hline 23 & 21 & 105 & 0.57499 & 0.123168 & -0.008001 & 0.022386 \\
\hline 24 & 105 & 105 & 0.36370 & 0.187748 & -0.010315 & 0.028440 \\
\hline 25 & 105 & 105 & 0.36370 & 0.187748 & -0.010315 & 0.028440 \\
\hline 26 & 107 & 107 & 0.34927 & 0.187640 & -0.010407 & 0.028660 \\
\hline 27 & 110 & 110 & 0.33457 & 0.187480 & -0.010544 & 0.028983 \\
\hline 28 & 30 & 120 & 0.30363 & 0.126312 & -0.008832 & 0.024461 \\
\hline 29 & 67 & 121 & 0.32780 & 0.152389 & -0.010053 & 0.027631 \\
\hline 30 & 72 & 121 & 0.40042 & 0.155847 & -0.010167 & 0.027925 \\
\hline 31 & 28 & 121 & 0.20316 & 0.124845 & -0.008758 & 0.024258 \\
\hline 32 & 88 & 122 & 0.30590 & 0.166027 & -0.010519 & 0.028816 \\
\hline 33 & 122 & 122 & 0.39079 & 0.186838 & -0.011070 & 0.030210 \\
\hline 34 & 128 & 128 & 0.34271 & 0.186518 & -0.011322 & 0.030788 \\
\hline 35 & 129 & 129 & 0.32798 & 0.186464 & -0.011363 & 0.030882 \\
\hline 36 & 25 & 140 & 0.18465 & 0.120759 & -0.009002 & 0.024725 \\
\hline 37 & 153 & 153 & 0.23087 & 0.185185 & -0.012301 & 0.032978 \\
\hline 38 & 36 & 155 & 0.15527 & 0.123779 & -0.009841 & 0.026765 \\
\hline 39 & 28 & 156 & 0.17473 & 0.120287 & -0.009476 & 0.025810 \\
\hline 40 & 162 & 162 & 0.25546 & 0.184707 & -0.012630 & 0.033692 \\
\hline 41 & 162 & 162 & 0.25546 & 0.184707 & -0.012630 & 0.033692 \\
\hline 42 & 164 & 164 & 0.23715 & 0.184601 & -0.012701 & 0.033846 \\
\hline
\end{tabular}

\begin{tabular}{|c|c|c|c|c|c|c|}
\hline 43 & 22 & 172 & 0.09767 & 0.118236 & -0.009395 & 0.025447 \\
\hline 44 & 183 & 130 & 0.30409 & 0.212919 & -0.012027 & 0.032516 \\
\hline 45 & 194 & 94 & 0.51533 & 0.251975 & -0.010957 & 0.030137 \\
\hline 46 & 195 & 195 & 0.20854 & 0.182957 & -0.013746 & 0.036039 \\
\hline 47 & 168 & 200 & 0.25894 & 0.171375 & -0.013534 & 0.035480 \\
\hline 48 & 222 & 123 & 0.40646 & 0.235889 & -0.012180 & 0.032948 \\
\hline 49 & 226 & 226 & 0.20903 & 0.181320 & -0.014690 & 0.037923 \\
\hline 50 & 243 & 243 & 0.15116 & 0.180425 & -0.015172 & 0.038849 \\
\hline 51 & 248 & 100 & 0.56437 & 0.271405 & -0.011607 & 0.031708 \\
\hline
\end{tabular}



lifetime data in presence of censored data

\begin{tabular}{|c|c|c|c|c|c|c|}
\hline 52 & 262 & 262 & 0.11632 & 0.179428 & -0.015685 & 0.039807 \\
\hline 53 & 265 & 210 & 0.25600 & 0.198997 & -0.014733 & 0.038198 \\
\hline 54 & 269 & 120 & 0.43193 & 0.258080 & -0.012449 & 0.033625 \\
\hline 55 & 276 & 81 & 0.91799 & 0.313273 & -0.011031 & 0.030383 \\
\hline 56 & 318 & 140 & 0.48086 & 0.257357 & -0.013401 & 0.035748 \\
\hline 57 & 20 & 348 & 0.34759 & 0.121786 & -0.011347 & 0.028620 \\
\hline 58 & 350 & 350 & 0.21901 & 0.174843 & -0.017778 & 0.043405 \\
\hline 59 & 32 & 360 & 0.26658 & 0.114101 & -0.012339 & 0.030880 \\
\hline 60 & 363 & 363 & 0.23332 & 0.174171 & -0.018054 & 0.043841 \\
\hline 61 & 371 & 184 & 0.31530 & 0.241115 & -0.014913 & 0.038884 \\
\hline 62 & 52 & 380 & 0.25512 & 0.111622 & -0.013517 & 0.033446 \\
\hline 63 & 390 & 390 & 0.27556 & 0.172778 & -0.018606 & 0.044685 \\
\hline 64 & 392 & 122 & 0.48407 & 0.298986 & -0.013257 & 0.035541 \\
\hline 65 & 393 & 100 & 0.62609 & 0.328974 & -0.012457 & 0.033763 \\
\hline 66 & 414 & 414 & 0.29934 & 0.171545 & -0.019073 & 0.045370 \\
\hline 67 & 417 & 417 & 0.26522 & 0.171391 & -0.019130 & 0.045452 \\
\hline 68 & 418 & 220 & 0.30683 & 0.232394 & -0.016015 & 0.041023 \\
\hline 69 & 431 & 431 & 0.25960 & 0.170675 & -0.019392 & 0.045822 \\
\hline 70 & 466 & 119 & 0.54467 & 0.324662 & -0.013505 & 0.036142 \\
\hline 71 & 469 & 90 & 0.71187 & 0.372329 & -0.012374 & 0.033602 \\
\hline 72 & 39 & 487 & 0.35246 & 0.111143 & -0.013777 & 0.032920 \\
\hline 73 & 487 & 76 & 1.20037 & 0.410450 & -0.011787 & 0.032223 \\
\hline 74 & 491 & 180 & 0.42200 & 0.272072 & -0.015460 & 0.040172 \\
\hline 75 & 515 & 515 & 0.40492 & 0.166406 & -0.020837 & 0.047702 \\
\hline 76 & 526 & 121 & 0.72952 & 0.338212 & -0.013828 & 0.036881 \\
\hline 77 & 547 & 130 & 0.79477 & 0.331862 & -0.014225 & 0.037744 \\
\hline 78 & 583 & 583 & 0.43867 & 0.162991 & -0.021873 & 0.048873 \\
\hline 79 & 641 & 641 & 0.40002 & 0.160108 & -0.022678 & 0.049677 \\
\hline 80 & 653 & 653 & 0.36001 & 0.159515 & -0.022837 & 0.049823 \\
\hline 81 & 677 & 150 & 0.58521 & 0.336584 & -0.015354 & 0.040176 \\
\hline 82 & 716 & 716 & 0.35579 & 0.156421 & -0.023629 & 0.050496 \\
\hline 83 & 732 & 732 & 0.31274 & 0.155641 & -0.023820 & 0.050643 \\
\hline 84 & 781 & 781 & 0.26807 & 0.153265 & -0.024381 & 0.051041 \\
\hline 85 & 845 & 845 & 0.22168 & 0.150192 & -0.025064 & 0.051450 \\
\hline 86 & 847 & 847 & 0.17346 & 0.150096 & -0.025085 & 0.051461 \\
\hline 87 & 848 & 155 & -0.03727 & 0.360602 & -0.016042 & 0.041666 \\
\hline 88 & 860 & 860 & 0.16111 & 0.149476 & -0.025217 & 0.051530 \\
\hline 89 & 29 & 932 & 0.02372 & 0.118366 & -0.015190 & 0.031854 \\
\hline 90 & 957 & 957 & 0.14771 & 0.144900 & -0.026142 & 0.051921 \\
\hline 91 & 1030 & 210 & 0.43184 & 0.332757 & -0.018093 & 0.045647 \\
\hline 92 & 1063 & 240 & 0.25501 & 0.314741 & -0.018901 & 0.047057 \\
\hline 93 & 1074 & 120 & 0.63670 & 0.445927 & -0.015352 & 0.040307 \\
\hline 94 & 1111 & 1111 & 0.21970 & 0.137805 & -0.027418 & 0.052168 \\
\hline 95 & 1136 & 140 & 0.67924 & 0.421245 & -0.016240 & 0.042171 \\
\hline 96 & 1156 & 180 & 0.53414 & 0.373881 & -0.017579 & 0.044800 \\
\hline 97 & 1182 & 112 & 0.71546 & 0.477327 & -0.015239 & 0.040067 \\
\hline
\end{tabular}


Jorge Alberto Achcar, Jose Rafael Tovar Cuevas, Fernando A. Moala

\begin{tabular}{|c|c|c|c|c|c|c|}
\hline 98 & 1199 & 91 & 0.93416 & 0.532028 & -0.014298 & 0.037983 \\
\hline 99 & 1238 & 250 & 0.42522 & 0.324452 & -0.019562 & 0.048312 \\
\hline 100 & 1258 & 120 & 0.99116 & 0.471168 & -0.015716 & 0.041093 \\
\hline 101 & 1279 & 1279 & 0.47604 & 0.130312 & -0.028588 & 0.052051 \\
\hline 102 & 1298 & 1298 & 0.41305 & 0.129481 & -0.028708 & 0.052018 \\
\hline 103 & 25 & 1324 & 0.33206 & 0.122792 & -0.015721 & 0.029908 \\
\hline 104 & 1330 & 96 & 1.17644 & 0.536794 & -0.014769 & 0.039023 \\
\hline 105 & 1356 & 1356 & 0.47604 & 0.126965 & -0.029060 & 0.051896 \\
\hline 106 & 1363 & 200 & 0.84522 & 0.374547 & -0.018587 & 0.046753 \\
\hline 107 & 1377 & 123 & 1.43709 & 0.479705 & -0.016050 & 0.041802 \\
\hline 108 & 1384 & 200 & 1.07223 & 0.376389 & -0.018629 & 0.046839 \\
\hline 109 & 1433 & 236 & 0.84625 & 0.350012 & -0.019657 & 0.048634 \\
\hline 110 & 1447 & 220 & 1.03570 & 0.363788 & -0.019286 & 0.048021 \\
\hline 111 & 1470 & 180 & 0.98839 & 0.404611 & -0.018212 & 0.046115 \\
\hline 112 & 1496 & 307 & 0.89459 & 0.310142 & -0.021316 & 0.051217 \\
\hline 113 & 1499 & 1499 & 1.02715 & 0.120897 & -0.029843 & 0.051477 \\
\hline 114 & 1527 & 1527 & 0.95526 & 0.119732 & -0.029984 & 0.051378 \\
\hline 115 & 1535 & 1535 & 0.87684 & 0.119400 & -0.030023 & 0.051348 \\
\hline 116 & 1562 & 1562 & 0.79096 & 0.118286 & -0.030154 & 0.051247 \\
\hline 117 & 1568 & 1568 & 0.69648 & 0.118039 & -0.030182 & 0.051224 \\
\hline 118 & 1602 & 139 & 1.50734 & 0.473532 & -0.017046 & 0.043869 \\
\hline 119 & 1631 & 150 & 1.15761 & 0.458208 & -0.017496 & 0.044773 \\
\hline 120 & 1674 & 1674 & 0.86505 & 0.113737 & -0.030659 & 0.050784 \\
\hline 121 & 1799 & 140 & 1.60573 & 0.488687 & -0.017376 & 0.044544 \\
\hline 122 & 1829 & 1829 & 0.91692 & 0.107640 & -0.031268 & 0.050049 \\
\hline 123 & 1843 & 1843 & 0.79097 & 0.107100 & -0.031319 & 0.049978 \\
\hline 124 & 1850 & 1850 & 0.64561 & 0.106831 & -0.031343 & 0.049942 \\
\hline 125 & 1857 & 260 & 0.60245 & 0.359609 & -0.020992 & 0.051053 \\
\hline 126 & 1870 & 230 & 1.01756 & 0.383764 & -0.020283 & 0.049923 \\
\hline 127 & 2024 & 180 & 1.40613 & 0.444725 & -0.019082 & 0.047864 \\
\hline 128 & 2133 & 250 & 1.40611 & 0.380850 & -0.021178 & 0.051493 \\
\hline 129 & 2140 & 220 & 1.62277 & 0.407215 & -0.020417 & 0.050252 \\
\hline 130 & 2204 & 2204 & 2.05062 & 0.093847 & -0.032388 & 0.047971 \\
\hline
\end{tabular}


